\title{
Complete mitochondrial genome sequences of the northern spotted owl (Strix occidentalis caurina) and the barred owl (Strix varia; Aves: Strigiformes: Strigidae) confirm the presence of a duplicated control region
}

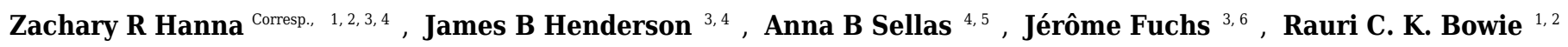
John P Dumbacher ${ }^{3,4}$

\footnotetext{
1 Museum of Vertebrate Zoology, University of California, Berkeley, Berkeley, California, United States of America

2 Department of Integrative Biology, University of California, Berkeley, Berkeley, California, United States of America

3 Department of Ornithology \& Mammalogy, California Academy of Sciences, San Francisco, California, United States of America

4 Center for Comparative Genomics, California Academy of Sciences, San Francisco, California, United States of America

5 Chan Zuckerberg Biohub, San Francisco, California, United States of America

6 UMR 7205 Institut de Systématique, Evolution, Biodiversité, CNRS, MNHN, UPMC, EPHE, Sorbonne Universités, Muséum National d'Histoire Naturelle, Paris, France

Corresponding Author: Zachary R Hanna Email address: zachanna@berkeley.edu
}

We report here the successful assembly of the complete mitochondrial genomes of the northern spotted owl (Strix occidentalis caurina) and the barred owl (S. varia). We utilized sequence data from two sequencing methodologies, Illumina paired-end sequence data with insert lengths ranging from approximately 250 nucleotides (nt) to 9,600 nt and read lengths from 100-375 nt and Sanger-derived sequences. We employed multiple assemblers and alignment methods to generate the final assemblies. The circular genomes of S. o. caurina and S. varia are comprised of 19,948 nt and 18,975 nt, respectively. Both code for two rRNAs, twenty-two tRNAs, and thirteen polypeptides. They both have duplicated control region sequences with complex repeat structures. We were not able to assemble the control regions solely using Illumina paired-end sequence data. By fully spanning the control regions, Sanger-derived sequences enabled accurate and complete assembly of these mitochondrial genomes. These are the first complete mitochondrial genome sequences of owls (Aves: Strigiformes) possessing duplicated control regions. We searched the nuclear genome of S. o. caurina for copies of mitochondrial genes and found at least nine separate stretches of nuclear copies of gene sequences originating in the mitochondrial genome (Numts). The Numts ranged from 226-19,522 nt in length and included copies of all mitochondrial genes except $t R N A^{\text {Pro }}, N D 6$, and $t R N A^{G / u}$. 
1 Complete mitochondrial genome sequences of the northern spotted owl (Strix occidentalis

2 caurina) and the barred owl (Strix varia; Aves: Strigiformes: Strigidae) confirm the presence of a

3 duplicated control region

4

5 Zachary R. Hanna, ${ }^{1,2,3,4,7}$ James B. Henderson, ${ }^{3,4}$ Anna B. Sellas, ${ }^{4,5}$ Jérôme Fuchs, ${ }^{3,6}$ Rauri C. K.

6 Bowie, ${ }^{1,2}$ John P. Dumbacher ${ }^{3,4}$

7

$8 \quad{ }^{1}$ Museum of Vertebrate Zoology, University of California, Berkeley, Berkeley, California,

9 United States of America

$10{ }^{2}$ Department of Integrative Biology, University of California, Berkeley, Berkeley, California,

11 United States of America

$12{ }^{3}$ Department of Ornithology \& Mammalogy, California Academy of Sciences, San Francisco,

13 California, United States of America

$14{ }^{4}$ Center for Comparative Genomics, California Academy of Sciences, San Francisco, California,

15 United States of America

$16{ }^{5}$ Chan Zuckerberg Biohub, San Francisco, California, United States of America

$17{ }^{6}$ UMR 7205 Institut de Systématique, Evolution, Biodiversité, CNRS, MNHN, UPMC, EPHE,

18 Sorbonne Universités, Muséum National d'Histoire Naturelle, Paris, France

$19{ }^{7}$ Corresponding Author:

20 Zachary Hanna

21 Email address: zachanna@berkeley.edu 


\section{Abstract}

We report here the successful assembly of the complete mitochondrial genomes of the

northern spotted owl (Strix occidentalis caurina) and the barred owl (S. varia). We utilized sequence data from two sequencing methodologies, Illumina paired-end sequence data with insert lengths ranging from approximately 250 nucleotides (nt) to 9,600 nt and read lengths from 100-375 nt and Sanger-derived sequences. We employed multiple assemblers and alignment methods to generate the final assemblies. The circular genomes of $S$. o. caurina and $S$. varia are comprised of 19,948 nt and 18,975 nt, respectively. Both code for two rRNAs, twenty-two tRNAs, and thirteen polypeptides. They both have duplicated control region sequences with complex repeat structures. We were not able to assemble the control regions solely using Illumina paired-end sequence data. By fully spanning the control regions, Sanger-derived sequences enabled accurate and complete assembly of these mitochondrial genomes. These are the first complete mitochondrial genome sequences of owls (Aves: Strigiformes) possessing duplicated control regions. We searched the nuclear genome of $S$. o. caurina for copies of mitochondrial genes and found at least nine separate stretches of nuclear copies of gene sequences originating in the mitochondrial genome (Numts). The Numts ranged from 226-19,522 nt in length and included copies of all mitochondrial genes except $t R N A^{P r o}, N D 6$, and $t R N A^{G l u}$.

\section{$\underline{\text { Introduction }}$}

The chicken (Gallus gallus) was the first avian species with a complete mitochondrial genome assembly (Desjardins \& Morais, 1990). Subsequently, researchers assembled the mitochondrial genomes of members of the Paleognathae (e.g., ostriches, emus, kiwis) and other members of the Galloanserae (ducks, chicken-like birds) and recovered the same gene order found in the mitochondrial genome of the chicken, which led to the conclusion that the 
46 mitochondrial genome of the chicken is representative of the ancestral avian gene order

47 (Desjardins \& Morais, 1990; Mindell, Sorenson \& Dimcheff, 1998a; Haddrath \& Baker, 2001;

48 Gibb et al., 2007). Almost a decade after publication of the chicken mitochondrial genome,

49 Mindell, Sorenson \& Dimcheff (1998a) described an alternative or, to use their terminology,

50 "novel" avian gene order from that of the chicken, which included a different positioning of

$51 t R N A^{P r o}, N D 6$, and $t R N A^{G l u}$ relative to the control region sequence as well as an additional

52 noncoding segment that they hypothesized was a degraded copy of the control region. A few

53 years later, researchers first described the presence of an intact, duplicated control region in the

54 mitochondrial genomes of Amazona parrots (Eberhard, Wright \& Bermingham, 2001) and the

55 common buzzard Buteo buteo (Haring et al., 2001).

56 Mindell, Sorenson \& Dimcheff (1998a) detected their novel avian gene order in the

57 mitochondrial genomes of taxa in multiple avian orders that spanned a significant portion of

58 Neoaves, but did not detect it in the single owl species that they studied, Otus asio (Mindell,

59 Sorenson \& Dimcheff, 1998a). However, further investigation of owl (Strigiformes)

60 mitochondrial genomes has revealed several surprises.

61 First, at least three wood owl species [Strix aluco, S. uralensis (Brito, 2005), and S. varia

62 (Barrowclough et al., 2011)] contain the novel mitochondrial gene order of Mindell, Sorenson \&

63 Dimcheff (1998a) as well as duplicate control regions. The use of a primer in $t R N A^{T h r}$ to amplify

64 a fragment of the control region suggests that the novel gene order is present in two additional

65 wood owl species, S. occidentalis (Barrowclough, Gutierrez \& Groth, 1999) and S. nebulosa

66 (Hull et al., 2010). However, the novel gene order was not reported as present in the

67 mitochondrial genome of S. leptogrammica (Liu, Zhou \& Gu, 2014). 
Second, some species of eagle-owls (genus $B u b o$ ) have a large control region (up to

69 3,800 nucleotides) relative to Strix, their putative sister genus (Fuchs et al., 2008; Wink et al., 70 2009), largely due to a tandem repeat structure in the 3' end of the control region (Omote et al.,

71 2013). Such control region tandem repeat blocks appear to be widespread in Strigidae (Xiao et

72 al., 2006; Omote et al., 2013). These results suggest that the structures of owl mitochondrial

73 genomes are surprisingly dynamic and in need of further investigation, particularly for species of

74 conservation concern for which portions of the control region are used in population genetic

75 studies (Barrowclough, Gutierrez \& Groth, 1999; Haig et al., 2004; Hull et al., 2010, 2014).

76 We here provide the complete mitochondrial genome sequence of both a northern spotted

77 owl (Strix occidentalis caurina) and barred owl (S. varia). The spotted owl (S. occidentalis) is a

78 large and charismatic denizen of dense forests whose range includes the Pacific coast of North

79 America from southwestern British Columbia to southern California and extends eastward into

80 the deserts of the Southwestern United States and southward to central Mexico. The range of the

81 northern spotted owl (S. o. caurina) subspecies includes the Pacific Northwest portion of the $S$.

82 occidentalis range from British Columbia south to the Golden Gate strait, California. The U.S.

83 Fish and Wildlife Service has listed S. o. caurina as "threatened" under the Endangered Species

84 Act since 1990.

85 The barred owl (S. varia), formerly native east of the Rocky Mountains (Mazur \& James,

86 2000), has extended its range into the western U.S. in the last 50-100 years and, from British

87 Columbia to southern California, has become broadly sympatric with the northern spotted owl in

88 the last 50 years. Barred and spotted owls hybridize and successfully backcross (Haig et al.,

89 2004; Kelly \& Forsman, 2004; Funk et al., 2007). Mitochondrial DNA sequencing has served as

90 a valuable tool in ascertaining the maternal lineage of western birds, especially in potential 
91 hybrids (Zink, 1994; Haig et al., 2004; Barrowclough et al., 2005; Ruegg, 2008; Krosby \&

92 Rohwer, 2009; Williford et al., 2014).

Population-level studies of the genetics of S. occidentalis and S. varia have mainly used two mitochondrial markers, a partial control region sequence (Barrowclough, Gutierrez \& Groth, 1999; Haig et al., 2004; Barrowclough et al., 2005) and cytochrome b (cyt b) (Haig et al., 2004), although a phylogeographic study of $S$. varia also utilized portions of ND6 and COIII (Barrowclough et al., 2011). The sequences of the complete genomes of the mitochondria of these two species will aid researchers in utilizing additional mitochondrial markers in population genetic studies of these owls.

It is well known that mitochondrial genes can transfer to the nuclear genome; such regions of the nuclear genome are sometimes called Numts (Lopez et al., 1994; Sorenson \& Quinn, 1998). As a high-quality nuclear genome of S. o. caurina is available (Hanna et al., 2017), we were able to explore the incidence of Numts within the nucleus and investigate which mitochondrial genes have most often transferred. Furthermore, by assessing divergence between mitochondrial genes and their descendent Numts, we ascertained the likelihood of them posing problems for phylogenetic and other types of studies.

\section{$\underline{\text { Methods }}$}

Strix occidentalis mitochondrial genome assembly

We sourced Strix occidentalis caurina DNA from a blood sample collected by a veterinarian from a captive adult female $S$. o. caurina at WildCare rehabilitation facility in San Rafael, California. Found as an abandoned nestling in Larkspur, Marin County, California, WildCare admitted the captive owl as patient card \# 849 on 5 June 2005 and named her Sequoia (sample preserved as CAS:ORN:98821; Table 1). 
115 sequence data from nine different genomic libraries constructed, sequenced, and processed as

116 described in Hanna et al. (2017). The raw sequences from sample CAS:ORN:98821 (Table 1) are

117 available from the NCBI Sequence Read Archive (SRA) (SRA run accessions SRR4011595,

118 SRR4011596, SRR4011597, SRR4011614, SRR4011615, SRR4011616, SRR4011617,

119 SRR4011618, SRR4011619, and SRR4011620). For our initial assembly, we used BLATq

120 version 1.02 (Henderson \& Hanna, 2016a), which was a modification of BLAT version 35 (Kent,

121 2002, 2012), to find Illumina reads that aligned to the Ninox novaeseelandiae mitochondrial

122 genome (GenBank Accession AY309457.1) (Harrison et al., 2004) (Supplementary Materials

123 (SM) 1.1.1) and extracted those matching reads using excerptByIds version 1.0.2 (Henderson \&

124 Hanna, 2016b) (SM 1.1.2). We then used SOAPdenovo2 version 2.04 (Luo et al., 2012) to

125 assemble those sequences (SM 1.1.3).

126 We used the web version of the NCBI BLAST+ version 2.2.29 tool BLASTN (Altschul

127 et al., 1990; Zhang et al., 2000; Morgulis et al., 2008; Camacho et al., 2009) to search the NCBI

128 nucleotide collection (Johnson et al., 2008; Boratyn et al., 2013; Benson et al., 2015;

129 NCBI Resource Coordinators, 2015) (NCBI-nt) to assess the completeness of the resulting

130 assembled continuous sequences (contigs) by aligning them to available mitochondrial genome

131 sequences (SM 1.1.4). We confirmed that we had assembled a contig with the genes for $t R N A^{P h e}$

132 through $c y t b$ to $t R N A^{T h r}$ that was approximately 18,000 nucleotides (nt) in length, but lacked the

133 complete control region sequence. We used GNU Grep version 2.16 (Free Software Foundation,

134 2014) to search the Illumina reads for matches to the assembled sequence of $t R N A^{P h e}$ or $t R N A^{T h r}$

135 (SM 1.1.5). We found three reads that spanned $t R N A^{\text {Phe }}$ and combined them using the Geneious

136 version 9.1.4 de novo assembler (Kearse et al., 2012; Biomatters, 2016) (SM 1.1.6). We then 
137 extended this assembled contig using a targeted assembly approach with the software PRICE

138 version 1.2 (Ruby, Bellare \& DeRisi, 2013; Ruby, 2014) (SM 1.1.7). This PRICE run produced

139 an improved and lengthened assembly after 31 cycles, but the assembly still lacked the complete

140 control region sequence.

141 We used BLATq version 1.0.2 to align Illumina sequences to the assembly output by

142 PRICE (SM 1.1.8) and extracted aligned reads using excerptByIds version 1.0.2 (SM 1.1.9). We

143 then performed another PRICE assembly with the same initial contig as before, but with the

144 extracted additional Illumina sequence data (SM 1.1.10). This run produced an assembly of one

145 contig of length 18,489 nt after 26 cycles.

146 We annotated this PRICE assembly using the MITOS WebServer version 605 (Bernt et

147 al., 2013) (SM 1.1.11), which confirmed that this assembly contained the genes for $t R N A^{P h e}$

148 through $c y t b$ to $t R N A^{T h r}$ followed by control region 1 (CR1), $t R N A^{\text {Pro }}$, ND6, $t R N A^{G l u}$, and control

149 region 2 (CR2). We searched for repetitive regions using Tandem Repeats Finder version 4.07b

150 (Benson, 1999, 2012) (SM 1.1.12).

151 In order to confirm the assemblies of both CR1 and CR2 with longer sequences that

152 could span the repetitive sections of these regions, we designed primers to gene sequences

153 outside of CR1 and CR2 and used Sanger sequencing to obtain verifying sequences across them.

154 We successfully amplified CR2 using a polymerase chain reaction (PCR) with primers 17589F

155 and 41R (Table 2), which primed in $t R N A^{G l u}$ and $t R N A^{P h e}$, respectively. We then sequenced both

156 ends of the PCR-amplified fragment using BigDye terminator chemistry (Applied Biosystems,

157 Foster City, Calif., U.S.A.) on an ABI 3130xl automated sequencer (Applied Biosystems, Foster

158 City, Calif., U.S.A.; SM 1.2.1). We also used primer $17572 \mathrm{~F}$, which primed in $t R N A^{G l u}$, and

159 primer 41R (Table 2) to successfully PCR-amplify a slightly longer fragment than above, which 
160 also included all of CR2, and then sequenced across the repetitive section of CR2 using internal

161 primers $18327 \mathrm{~F}$ and 19911R (Table 2), which primed outside of the repetitive region (SM 1.2.2).

162 We edited the sequences using Geneious version 9.1.4 (Kearse et al., 2012; Biomatters,

1632016 ) and then used the Geneious mapper to align the sequences to the 19,946 nt preliminary

164 mitochondrial genome assembly (SM 1.2.3). These Sanger-derived sequences confirmed that

165 there were nine complete repetitions of a $78 \mathrm{nt}$ motif in CR2 and extended the assembly length to $16619,948 \mathrm{nt}$.

167 Similarly, we confirmed the CR1 sequence with Sanger-derived sequence data by first

168 PCR-amplifying CR1 with primers cytb-F1 and 17122R (Table 2), which primed in $c y t b$ and

169 ND6, respectively (SM 1.2.4). We visualized the PCR products on a 1\% agarose gel, which

170 revealed two PCR products approximately 2,250 and 3,500 nt in length. We re-ran the PCR and

171 gel visualization to confirm this result, which was consistent. We then excised each band from a

$1721 \%$ low melting point agarose gel, performed gel purification using a Zymoclean Gel DNA

173 Recovery Kit (Zymo Research, Irvine, Calif., U.S.A.), and sequenced the purified fragments

174 using the original external primers as well as the internal primers CR1-F1, CR1-F1-RC, CR1-R2,

175 CR1-R2-RC, and N1 (Barrowclough, Gutierrez \& Groth, 1999) (Table 2) with BigDye

176 terminator chemistry on an ABI 3130xl automated sequencer. We edited the sequences using

177 Geneious version 9.1.4 and then used the Geneious de novo assembler and mapper to assemble

178 the sequences and then align them to the $19,948 \mathrm{nt}$ preliminary mitochondrial genome assembly.

179 We were able to assemble the entirety of the smaller PCR product, but we were unable to

180 completely assemble the CR1 repetitive region in the larger PCR product. Thus, our

181 mitochondrial genome assembly contains the CR1 sequence obtained from the smaller PCR

182 product. The assembly length was then 19,889 nt as the Sanger-confirmed CR1 sequence 
183 contained a shorter repetitive region than we assembled with the shorter Illumina sequences. The

184 length of the CR1 repetitive region in the Illumina-sequence-only assembly was also different

185 from the length we expected in the larger PCR product.

186 In order to use all of the available Illumina sequence data to verify our mitochondrial

187 genome assembly, we took the draft whole genome assembly of $S$. o. caurina (Hanna et al.,

188 2017) and replaced scaffold-3674, which was the incomplete assembly of the mitochondrial

189 genome, with the 19,889 nt mitochondrial genome assembly from our targeted assembly

190 methodology (SM 1.3.1).

191 We aligned all filtered Illumina sequences to this new draft reference genome using bwa

192 version 0.7.13-r1126 (Li, 2013a) and then merged, sorted, and marked duplicate reads using

193 Picard version 2.2.4 (http://broadinstitute.github.io/picard) (SM 1.3.2). We filtered the alignment

194 file to only retain alignments to the preliminary targeted mitochondrial genome assembly using

195 Samtools version 1.3 with HTSlib 1.3.1 (Li et al., 2009, 2015). We then used Samtools and GNU

196 Awk (GAWK) version 4.0.1 (Free Software Foundation, 2012) to filter the alignments (SM

197 1.3.3-1.3.4). We next visualized the alignment across the reference sequence in Geneious version

198 9.1.4 to confirm that the sequence evidence matched our assembly (SM 1.3.5).

199 We annotated the final assembly using the MITOS WebServer version 806 (Bernt et al.,

200 2013) (SM 1.4.1) followed by manual inspection of the coding loci and comparison with

201 predicted open reading frames and sequences from Gallus gallus [GenBank Accessions

202 NC_001323 (Desjardins \& Morais, 1990) and AB086102.1 (Wada et al., 2004)] in Geneious

203 version 9.1.4. We annotated the repetitive regions using the web version of Tandem Repeats

204 Finder version 4.09 (Benson, 1999, 2016) (SM 1.4.2). We used bioawk version 1.0 (Li, 2013b)

205 and GAWK version 4.0.1 to find goose hairpin sequences in CR1 and CR2 (SM 1.4.3). We 
206 compared the sequences of the annotated genes in our final mitochondrial genome assembly with

207 those of the incomplete mitochondrial genome assembly that was output as a byproduct of the $S$.

208 o. caurina whole nuclear genome assembly (Hanna et al., 2017) in order to evaluate the efficacy

209 of the nuclear genome assembler in assembling mitochondrial genes. We aligned all of the

210 nucleotide sequences of the genes in the final mitochondrial genome against a database of the

211 scaffold-3674 gene nucleotide sequences using NCBI BLAST+ version 2.4.0 tool BLASTN

212 (Altschul et al., 1990; Zhang et al., 2000; Morgulis et al., 2008; Camacho et al., 2009) (SM

213 1.4.4).

214 In order to visualize the binding sites of the primers that we developed to PCR-amplify

215 CR1 and CR2 as well as the primers used by Barrowclough, Gutierrez \& Groth (1999) to PCR-

216 amplify a portion of CR1 we used Geneious version 9.1.4 (SM 1.4.5). We assessed the similarity

217 of CR1 and CR2 by performing a multiple alignment using the Geneious version 9.1.4

218 implementation of MUSCLE version 3.8.425 (Edgar, 2004) (SM 1.4.6). In order to assess

219 whether published control region sequences of related species are more similar to CR1 or CR2,

220 we used the web version of NCBI's BLAST+ version 2.5.0 tool BLASTN (Altschul et al., 1990;

221 Zhang et al., 2000; Morgulis et al., 2008; Camacho et al., 2009) to search NCBI-nt for sequences

222 similar to CR1 and CR2 (SM 1.4.7). As a result of these searches, we aligned the primers used

223 by Omote et al. (2013) to PCR-amplify the control region in Strix uralensis in their study to our

224 final S. o. caurina assembly using Geneious version 9.1.4 (SM 1.4.8).

225 Nuclear pseudogenation of Strix occidentalis mitochondrial genes

226 In order to examine the incidence of genetic transfer from mitochondria to the nucleus,

227 we examined the draft nuclear genome assembly for evidence of nuclear pseudogenes or nuclear

228 copies of mitochondrial genes (Numts) (Lopez et al., 1994), in the S. o. caurina draft nuclear 
229 genome assembly (Hanna et al., 2017). We aligned the final S. o. caurina mitochondrial genome

230 assembly to the draft nuclear genome assembly using the NCBI BLAST+ version 2.4.0 tool

231 BLASTN (SM 1.5.1) using the default threshold Expect value (E-value) of 10. We then used

232 GAWK version 4.0.1 to remove all alignments to scaffold-3674, which was the assembly of the

233 mitochondrial genome in the draft nuclear genome assembly. We visually inspected the results to

234 insure that all alignments were of reasonable length and that all E-values were $<0.0001$ (De Wit

235 et al., 2012). Indeed, all alignments exceeded $100 \mathrm{nt}$ and all E-values were $<1 \times 10^{-25}$. We next

236 used GAWK version 4.0.1 to reformat the BLAST output into a Browser Extensible Data (BED)

237 formatted file (SM 1.5.3). In order to determine the mitochondrial genes spanned by each Numt,

238 we used BEDTools version 2.26.0 (Quinlan \& Hall, 2010) to produce a BED file of the

239 intersection of the BED-formatted BLAST output with the BED file output from the MITOS

240 annotation of the final mitochondrial genome assembly (SM 1.5.4).

241 Strix varia mitochondrial genome assembly

242 In order to assess the divergence between S. occidentalis and S. varia across all genes of

243 the mitochondrial genome, we constructed a complete $S$. varia mitochondrial genome assembly.

244 We did this by utilizing available whole-genome Illumina data from two $S$. varia individuals

245 collected outside of the zone of contact of S. varia and S. o. caurina (Haig et al., 2004). The

246 main set of $S$. varia whole-genome Illumina data originated from sequencing of a tissue sample

247 collected in Hamilton County, Ohio, United States of America (CMC:ORNI-T:B41533; Table

248 1), hereafter "CMCB41533". The paired-end Illumina sequence data was from a genomic library

249 constructed, sequenced, and the data processed as described in Hanna et al. (2017). The raw

250 sequences are available from NCBI (SRA run accessions SRR5428115, SRR5428116, and

251 SRR5428117). 


\section{3}

254

255

256

257

258

259

260

261

262

263

264

265

266

America (CAS:ORN:95964; Table 1), hereafter “CAS95964”. Sequence data from this

individual informed the assembly process, but none of these data are included in the final $S$.

varia mitochondrial genome assembly (SM 1.6.1). The raw sequences are available from NCBI

(SRA run accession SRRXXXXXXX). We performed adapter and quality trimming of these

sequence data using Trimmomatic version 0.30 (Bolger, Lohse \& Usadel, 2014) (SM 1.6.2). For

use in only the SOAPdenovo2 assembly, we trimmed the sequences using a different set of

parameters and performed error-correction of the sequences using SOAPec version 2.01 (Luo et al., 2012) (SM 1.6.3).

We constructed the complete $S$. varia mitochondrial genome of sample CMCB41533 by

building a succession of assemblies that contributed information to the final assembly from which we extracted the gene sequences. We used partial mitochondrial assemblies of sample CAS95964 to inform the assembly process, but, as we had more sequence data for sample CMCB41533, we chose to only produce a final genome assembly for this sample to compare with that of $S$. o. caurina.

We used two contigs (ContigInput1 and ContigInput2) as the starting material for our final CMCB41533 S. varia assembly. In order to generate ContigInput1, we used bwa version 0.7.13-r1126 to align all of the trimmed CMCB41533 paired read 1 and 2 sequences to a reference sequence that included the draft $S$. o. caurina whole nuclear genome along with our final mitochondrial genome assembly (SM 1.9.1). We then merged the paired-end and unpaired read alignments, sorted the reads, and marked duplicate reads using Picard version 2.2.4 (SM 1.9.2). 
We filtered the alignment file to only retain alignments to the final mitochondrial genome

275

276

277

278

279

280

281

282

283

284

285

286

287

288

289

290

291

292

293

294 295

assembly using Samtools version 1.3 with HTSlib 1.3.1 (Li et al., 2009, 2015). We then used

Samtools and GAWK version 4.0.1 to filter out duplicate reads, low quality alignments, secondary alignments, and alignments where both reads of a pair did not align to the

mitochondrial assembly (SM 1.9.2-1.9.3). We next visualized the alignment across the reference sequence in Geneious version 9.1.4 and generated a consensus sequence from the alignment (SM 1.9.4). We extracted three sequences from this consensus sequence based on the S. o. caurina mitochondrial genome annotations and then used these extracted sequences as three separate seed contigs in an assembly using PRICE version 1.2 (SM 1.9.5). This run produced one contig (ContigInput1) of length 9,690 nt after 16 cycles.

The series of assemblies that resulted in ContigInput2, an input to our final $S$. varia assembly, involved first using SOAPdenovo2 version 2.04 to assemble all of the trimmed, errorcorrected CAS95964 sequences (SM 1.10.1). We extended the output 15,019 nt contig using PRICE version 1.2 (SM 1.10.2). After seven cycles, this run produced an assembly of one contig of length $16,652 \mathrm{nt}$, which included the sequence for $t R N A^{\text {Phe }}$ through $t R N A^{T h r}$ and part of CR1. We used this CAS95964 contig to seed a more complete assembly using PRICE version 1.2 with the larger CMCB41533 Illumina sequence dataset (SM 1.11.1). After four cycles, this assembly produced one contig of length $17,073 \mathrm{nt}$, which we will refer to as "ContigInput2" below.

We performed a final assembly using PRICE version 1.2 and the 9,690 nt ContigInput1 and the 17,073 nt ContigInput2 as the initial contigs (SM 1.12.1). After two cycles, this assembly produced one contig of length 19,589 nt. We then used Sanger sequencing to confirm the sequences of CR1 and CR2. 
We PCR-amplified CR1 with primers cytb-F1 and 17122R (Table 2), which primed in cyt

$297 b$ and ND6, respectively (SM 1.12.2). We then sequenced the fragment using the original

298 external primers as well as the internal primers CR1-F1, CR1-F1-RC, CR1-R2, CR1-R2-RC, and

299 N1 (Barrowclough, Gutierrez \& Groth, 1999) (Table 2). We PCR-amplified CR2 with primers

300 ND6-ext1F and 12S-ext1R (Table 2), which primed in ND6 and 12S, respectively (SM 1.12.3).

301 We then sequenced the PCR-amplified fragment using the original external primers as well as

302 the internal primers final-CR2F, 18547F, 19088R, and 19088R-RC. We performed all

303 sequencing using BigDye terminator chemistry (Applied Biosystems, Foster City, Calif., U.S.A.)

304 on an ABI 3130xl automated sequencer (Applied Biosystems, Foster City, Calif., U.S.A.).

305 We edited the sequences using Geneious version 9.1.4 and then used the Geneious de

306 novo assembler and mapper to assemble the sequences and then align them to the $19,589 \mathrm{nt}$

307 preliminary mitochondrial genome assembly. These Sanger-derived sequences confirmed that

308 the preliminary assembly was inaccurate in the control regions and reduced the total length to a

309 final size of 18,975 nt. We annotated the assembly using the MITOS WebServer version 605

310 (SM 1.12.4) followed by manual inspection of the coding loci and comparison with predicted

311 open reading frames and sequences from Gallus gallus [GenBank Accessions NC_001323

312 (Desjardins \& Morais, 1990) and AB086102.1 (Wada et al., 2004)] in Geneious version 9.1.4.

313 We annotated the repetitive regions using the web version of Tandem Repeats Finder version

314 4.09 (Benson, 1999, 2016) (SM 1.4.2). We used bioawk version 1.0 (Li, 2013b) and GAWK

315 version 4.0.1 to find goose hairpin sequences in CR1 and CR2 (SM 1.4.3).

316 Comparison of Strix occidentalis and Strix varia mitochondrial genes

317 In order to compare mitochondrial gene sequences of S. occidentalis and S. varia, we

318 extracted the nucleotide sequence for all non-tRNA genes (stop codons excluded) from our final 
319 S. o. caurina and S. varia assemblies. We aligned them using MAFFT version 7.305b (Katoh et

320 al., 2002; Katoh \& Standley, 2013; Katoh, 2016) (SM 1.13.1). We verified the alignments by eye

321 and then used trimAl version 1.4.rev15 (Capella-Gutiérrez, Silla-Martínez \& Gabaldón, 2009;

322 Capella-Gutiérrez \& Gabaldón, 2013) to convert the alignments to MEGA format (Kumar,

323 Tamura \& Nei, 1994; Kumar, Stecher \& Tamura, 2016) (SM 1.13.2). We then used MEGA

324 version 7.0.18 (Kumar, Stecher \& Tamura, 2016) to calculate the p-distance (SM 1.13.3) and the

325 corrected pairwise distance (Tamura \& Nei, 1993) (SM 1.13.4) between S. o. caurina and S.

326 varia for each gene. We calculated a weighted average pairwise distance across all of the genes

327 (SM 1.13.5).

328 Avian mitochondrial gene order comparisons

329 We downloaded the mitochondrial genome sequences of Gallus gallus (GenBank

330 Accession NC_001323.1) (Desjardins \& Morais, 1990), Melopsittacus undulatus (GenBank

331 Accession NC_009134.1) (Guan, Xu \& Smith, 2016), Falco peregrinus (GenBank Accession

332 NC_000878.1) (Mindell et al., 1997; Mindell, Sorenson \& Dimcheff, 1998a; Mindell et al.,

333 1999), Bubo bubo (GenBank Accession AB918148.1) (Hengjiu et al., 2016), Ninox

334 novaeseelandiae (GenBank Accession AY309457.1) (Harrison et al., 2004), Tyto alba (GenBank

335 Accession EU410491.1) (Pratt et al., 2009), Strix leptogrammica (GenBank Accession

336 KC953095.1) (Liu, Zhou \& Gu, 2014), Glaucidium brodiei (GenBank Accession KP684122.1)

337 (Sun et al., 2016), and Asio flammeus (GenBank Accession KP889214.1) (Zhang et al., 2016),

338 which were all submitted as complete genomes apart from Tyto alba, which was submitted as a

339 partial genome. The Gallus gallus mitochondrion represented the ancestral avian order

340 (Desjardins \& Morais, 1990; Mindell, Sorenson \& Dimcheff, 1998a; Haddrath \& Baker, 2001;

341 Gibb et al., 2007). The mitochondrial gene order of Falco peregrinus was illustrative of the 
342 novel gene order first described by Mindell, Sorenson \& Dimcheff (1998a) with a remnant CR2

343 (Gibb et al., 2007) while the mitochondrial gene order of Melopsittacus undulatus exemplified

344 an intact, duplicated control region first described in Psittaciformes by Eberhard, Wright \&

345 Bermingham (2001). We visualized the mitochondrial genome sequences and the accompanying

346 annotations using Geneious version 9.1.4. For a coarse assessment of gene similarity, we next

347 used the Geneious version 9.1.4 implementation of MUSCLE version 3.8.425 in order to align

348 all of the owl (Aves: Strigiformes) mitochondrial genomes as well as to align the $S$.

349 leptogrammica mitochondrial genome with our S. o. caurina and S. varia assemblies.

350 Results

$351 \quad$ We deposited the complete mitochondrial genome sequences of Strix occidentalis

352 caurina sample CAS:ORN:98821 and Strix varia sample CMC:ORNI-T:B41533 as NCBI

353 GenBank Accessions MF431746 and MF431745, respectively. The lengths of the final S. $o$.

354 caurina and S. varia mitochondrial genome assemblies were $19,889 \mathrm{nt}$ and $18,975 \mathrm{nt}$,

355 respectively. As for all typical avian mitochondrial genomes, they are circular and code for 2

356 rRNAs, 22 tRNAs, and 13 polypeptides (Figure SM1 and Figure SM2). The annotations

357 produced by MITOS identified a $1 \mathrm{nt}$ gap that split $N D 3$, which is consistent with the

358 untranslated nucleotide and translational frameshift seen in ND3 in some other bird species

359 (Mindell, Sorenson \& Dimcheff, 1998b), including owls (Strigiformes) (Fuchs et al., 2008).

360 Both the S. o. caurina and S. varia mitochondrial genomes contain a duplicated control

361 region (Figure 1). In both genomes, CR1 and CR2 each include a C-rich sequence near the 5'

362 end, the goose hairpin (Quinn \& Wilson, 1993), which is identical across the two species and

363 across CR1 and CR2. The S. o. caurina CR1 contains a $70 \mathrm{nt}$ motif repeated 6.8 times near the 3' 
364 end while CR2 includes two sets of tandem repeats near the 3 ' end of the region, a 70 nt motif

365 repeated 4 times followed by 9.5 repetitions of a $78 \mathrm{nt}$ motif (Table 3 ).

366 The S. o. caurina CR1 and CR2 share a conserved central block of 1,222 nt with only two

367 mismatches between CR1 and CR2 (Figure 2). This conserved block includes $202 \mathrm{nt}$ of the 5'

368 portion of the repetitive regions. The $S$. varia $\mathrm{CR} 1$ and $\mathrm{CR} 2$ share a conserved $1,041 \mathrm{nt}$ central

369 sequence stretch containing five mismatches. In CR1, this conserved block begins in the $3^{\prime}$ $57 \mathrm{nt}$

370 of the CR1 repetitive region, but in CR2 it does not extend into the repetitive region. The 5' and

371 ' regions surrounding the conserved central blocks of the control regions in both $S$. o. caurina

372 and $S$. varia are more divergent from each other.

373 We obtained an alignment ( $88.37 \%$ identity) of $1,429 \mathrm{nt}$ from the 5 ' ends of the $S . o$.

374 caurina and S. varia $\mathrm{CR} 1$ sequences, but it included fifteen gaps (Figure 3 ). In contrast, the more

375 3' repetitive sections of the S. o. caurina and S. varia CR1 sequences yielded an uninformative

376 alignment with numerous, long gap regions. Similarly to CR1, the 5' ends of the S. o. caurina

377 and S. varia CR2 sequences aligned well (90.62\% identity), yielding a 1,300 nt alignment that

378 included four gaps. However, the alignment of the 3' region of the CR2 sequences was

379 uninformative with numerous, long gaps due to conflicts between the $78 \mathrm{nt}$ motif repetitive

380 regions of the two CR2 sequences. We found no evidence of mitochondrial pseudogenes in the

381 control region sequences of either S. o. caurina or S. varia.

382 Across all of the 35 genes that were present in the previous, incomplete $S$. o. caurina

383 assembly that was produced as a byproduct of the assembly of the S. o. caurina whole nuclear

384 genome (Hanna et al., 2017), we only found one mismatch with our complete assembly, which

385 occurred between the two ND1 sequences. This assembly improves upon the previous version by

386 providing the complete sequences of $N D 6, t R N A^{\text {Pro }}$, and the two control regions. 

species, the 5' end of CR1 borders $t R N A^{T h r}$ and the 3' end is adjacent to $t R N A^{P r o}$, then $N D 6$, and then $t R N A^{G l u}$ (Figure 1). The initial 1,104 nt of the $S$. o. caurina CR1 are identical to a $S . o$. caurina partial control region sequence (GenBank Accession AY833630.1) (Barrowclough et al., 2005). All of the top 100 matches of the BLASTN searches of the $S$. o. caurina CR1 to NCBI-nt were to either $S$. occidentalis or $S$. varia control region sequences deposited by other researchers, 393 as we expected.

CR2 follows $t R N A^{G l u}$ and is 2,319 nt in length in $S$. o. caurina and 1,719 nt long in $S$. varia. The initial $549 \mathrm{nt}$ of the $S$. o. caurina CR2 matches the beginning of the D-loop sequence of an annotated complete genome of a Bubo bubo mitochondrion (GenBank Accession AB918148.1) (Hengjiu et al., 2016). One of the top 100 matches of the BLASTN searches of the S. o. caurina CR1 to NCBI-nt, which had the highest total score $(2,177)$ and query coverage (96\% versus $36-41 \%$ for the other matches) of the top 100 matches, was to a $S$. uralensis control region sequence (GenBank Accession AB743794.1) (Omote et al., 2013). The majority of the primers used by Omote et al. (2013) to PCR-amplify the control region in S. uralensis align within and around the $S$. o. caurina CR2. Four of the control-region-specific primers align to the middle of CR2 in our S. o. caurina sequence, which is identical to the middle of the $S$. o. caurina 404 CR1 sequence. Perhaps most crucially, the primer L16728 aligns in the forward direction in $t R N A^{G l u}$ such that it would amplify CR2, if present in the species. As we mentioned in the methodology, our PCR-amplification of the S. o. caurina CR1 using primers that spanned from $c y t b$ to ND6 yielded two products approximately 2,250 and 3,500 nt in length (Figure SM3). The sequences of these fragments were identical in the $c y t b$ 
410 region at the 3' end of CR1. We were only able to obtain sequence spanning the entirety of this

411 repetitive region in the $2,250 \mathrm{nt}$ fragment. This was largely due to the fact that the $3,500 \mathrm{nt}$

412 fragment, in addition to the $70 \mathrm{nt}$ motif repetitive section observed in the sequence of the 2,250

413 nt fragment, contained another repetitive region on the $t R N A^{P r o}$ side of the $70 \mathrm{nt}$ motif region

414 with at least 13.1 copies of a 67 nt motif. We did not find any copies of $t R N A^{\text {Pro }}$ or ND6 in the $S$.

415 o. caurina nuclear genome, but we did find nuclear copies of $c y t b$ and $t R N A^{T h r}$. With $N D 6$

416 absent from the nuclear genome, PCR-amplification using primers in $c y t b$ and ND6 should only

417 generate mitochondrial genome fragments. Additionally, the $c y t b$ and $t R N A^{T h r}$ sequence in both

418 the 2,250 nt and 3,500 nt fragments did not match the nuclear genome copies of these genes. In

419 summary, we believe that both of these fragments were mitochondrial in origin and this evidence

420 suggests that at least two different versions of the mitochondrial genome were present in this $S$.

421 o. caurina individual.

422

The annotations of the mitochondrial genome sequences of the owls (Aves: Strigiformes)

Tyto alba, Ninox novaeseelandiae, Strix leptogrammica, Glaucidium brodiei, and Asio flammeus

424 indicate that those owls all share the same mitochondrial gene order as Gallus gallus, the

425 ancestral avian mitochondrial gene order (Desjardins \& Morais, 1990; Mindell, Sorenson \&

426 Dimcheff, 1998a; Haddrath \& Baker, 2001) (Figure 1). Our alignment of the S. leptogrammica

427 mitochondrial genome to the mitochondrial genomes of other owls, including our S. o. caurina

428 and $S$. varia assemblies, resulted in a poor, gap-filled alignment of the genes from the second

429 half of the S. leptogrammica cyt $b$ sequence through $N D 6$ to $t R N A^{\text {Phe }}$. We could not obtain a

430 reasonable alignment of the last $210 \mathrm{nt}$ of the $S$. leptogrammica D-loop adjacent to the $t R N A^{P h e}$

431 sequence to our $S$. o. caurina and $S$. varia assemblies or to the mitochondrial genomes of any of

432 the other Strigiformes whose sequences we examined. Additionally, alignment of the $S$. 
433 leptogrammica mitochondrial genome with our $S$. o. caurina and $S$. varia assemblies yielded an

434 ND5 alignment with seven gaps and numerous mismatches $(85.60 \%$ and $84.82 \%$ identity to $S$. $o$.

435 caurina and S. varia, respectively). Together, these results suggest that the S. leptogrammica

436 sequence potentially contains significant errors in the sequences of the genes from ND5 through 437 ND6 to $t R N A^{\text {Phe }}$.

438 We found 29,520 nt of Numt sequences in the draft S. o. caurina nuclear genome

439 assembly spanning nine Numts (Table 4). The Numts ranged in length from 226-19,522 nt and

440 had an average length of 3,280 nt. The Numts provided evidence of nuclear copies of all

441 mitochondrial genes, except $t R N A^{P r o}, N D 6$, and $t R N A^{G l u}$, the three genes between CR1 and CR2.

442 Numt \#9 (Table 4) aligns to both CR1 and CR2 with the alignments extending into the conserved

443 block shared by the control regions. The portion of genome scaffold-294 aligned to CR 2 for this

444 Numt is $519 \mathrm{nt}$, whereas the length aligned to CR1 is $592 \mathrm{nt}$. As we could not be sure of which

445 control region was incorporated into the nuclear genome, we have provided information for both 446 alignments and derived the length of the Numt from the alignment to CR1 (Table 4).

Strix occidentalis caurina and S. varia display an average of $10.74 \%(8.68 \%$ uncorrected

448 p-distance) base substitutions per site across the 2 rRNA genes and 13 polypeptide genes (the non-tRNA mitochondrial genes) (Table 5). The lowest number of base substitutions per site

450 occurs within $16 S$ and the highest within ATP8 (Table 5).

\section{Discussion}

452 Sequences of most mitochondrial genes can often be recovered from high-throughput

453 short-read sequencing data if genome complexity is not too great. Algorithms using short-read

454 data have more difficulty assembling low-complexity or repetitive regions due to an inability to

455 span these regions. Thus, assembly of complete mitochondrial genome sequences can be more 
456 difficult when such genomes include regions of low-complexity. The sequence of the avian

457 control region can both contain blocks of tandem repeats (Omote et al., 2013) and be duplicated

458 (Eberhard, Wright \& Bermingham, 2001; Haring et al., 2001). Moreover, the presence of

459 multiple controls regions that are similar or identical, which has been observed in snakes

460 (Kumazawa et al., 1996), can cause problems with assembly. In such situations, additional types

461 of sequencing data that complement short-read data may be necessary in order to obtain an

462 accurate and complete assembly of the mitochondrial genome. This proved to be the case in our

463 study where the longer Sanger-derived sequence data were crucial in obtaining the complete

464 sequence of the lengthy, repeat-rich control regions in S. o. caurina and S. varia. Although Brito

465 (2005) and Barrowclough et al. (2011) inferred the presence of a duplicated control region

466 structure in the mitochondrial genomes of at least three wood owl species, Strix aluco, $S$.

467 uralensis, and S. varia, they did not sequence complete mitochondrial genomes. They likely

468 deduced that a duplication was present from the appearance of multiple bands on agarose gels

469 resulting from PCR-amplification of portions of the mitochondrial control region. Here we

470 describe the first complete genome sequences of the mitochondrion of an owl (Aves:

471 Strigiformes) with a duplicate control region.

472 The mitochondrial genomes of S. o. caurina and S. varia exhibit the novel avian gene

473 order first described by Mindell, Sorenson \& Dimcheff (1998a) for several bird orders, but not

474 reported by them as present in the owl Otus asio. As mentioned above, this duplicated control

475 region structure and novel gene order has previously been reported in the mitochondrial genome

476 of S. varia (Barrowclough et al., 2011) and the congeners S. aluco and S. uralensis (Brito, 2005).

477 The novel gene order was previously implied for S. occidentalis by the placement of primer N1

478 in $t R N A^{T h r}$ by Barrowclough, Gutierrez \& Groth (1999) to PCR-amplify the control region (CR1) 
479 fragment used in their study. Hull et al. (2010) also used the Barrowclough, Gutierrez \& Groth 480 (1999) N1 primer to PCR-amplify the control region in their study of S. nebulosa, so we can 481 infer that the S. nebulosa mitochondrion also possesses the Mindell, Sorenson \& Dimcheff 482 (1998a) novel gene order. Notably, this mitochondrial gene order was not reported as present in 483 S. leptogrammica (Liu, Zhou \& Gu, 2014). However, our alignments of this mitochondrial 484 genome to our S. o. caurina and S. varia sequences as well as the sequences of other owl 485 mitochondrial genomes indicated problems with the $S$. leptogrammica sequence from $c y t b$ 486 through $N D 6$ to $t R N A^{\text {Phe }}$. If we then leave aside the S. leptogrammica sequence, available 487 evidence suggests that the novel gene order and duplicate control region structure is present 488 across the genus Strix. fragment of the control region (CR1) in S. occidentalis have been used extensively in additional genetic studies of owl species (Haig et al., 2004; Brito, 2005; Marthinsen et al., 2009; Hull et al., 2010; Barrowclough et al., 2011; Hausknecht et al., 2014). The Barrowclough, Gutierrez \&

Groth (1999) control region primers D16 (the most 3' of their primers) and D20 (more 5' relative 494 to primer D16) align to a region conserved between CR1 and CR2, although the length of the 495 distance from the binding site of primer N1 in $t R N A^{T h r}$ to the CR2 sites of primers D16 and D20 $496(3,742 \mathrm{nt}$ and $3,392 \mathrm{nt}$, respectively, in our S. o. caurina assembly) likely reduces the probability 497 of this second primer binding site causing problems in the PCR-amplification of the CR1 498 fragment.

499 The second control region appears intact, not degraded as found in some other avian taxa 500 (Mindell, Sorenson \& Dimcheff, 1998a). This gene order corresponds to the "Type D Duplicate 501 CR gene order" of Gibb et al. (2007) and the "Duplicate CR gene order I" of Eberhard \& Wright 
502 (2016). The goose-hairpin structure is typically found near the beginning of the control region in

503 avian mitochondria (Marshall \& Baker, 1997; Randi \& Lucchini, 1998; Bensch \& H, 2000) and,

504 in agreement with what we found, it appears in the beginning of the intact, duplicated control

505 region sequences in the genomes of Amazona (Eberhard, Wright \& Bermingham, 2001) and

506 additional parrot mitochondria (Eberhard \& Wright, 2016).

507 The lengths of the S. o. caurina CR1 and CR2 (2,021 nt and 2,319 nt, respectively) and of 508 the $S$. varia CR1 and CR2 (1,686 nt and 1,719 nt, respectively) are all shorter than the length 509 reported for the control regions of some species in the sister genus of owls, Bubo (Wink et al., 510 2009), which have lengths up to approximately $3,800 \mathrm{nt}$ due to tandem repeats in the 3 ' end of

511 the control region (Omote et al., 2013). Similar tandem repeat blocks occur in the control regions

512 of several other owl species in the family Strigidae (Xiao et al., 2006; Omote et al., 2013). The

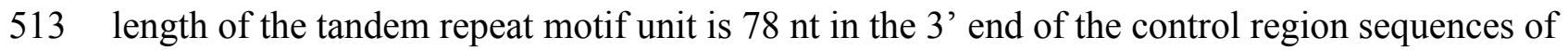

514 Bubo blakistoni, Bubo virginianus, Strix uralensis (Omote et al., 2013), and Strix aluco (Xiao et

515 al., 2006); $78 \mathrm{nt}$ is also the length of the motif in the longest tandem repeat block in both the $S . o$.

516 caurina and S. varia CR2 (Table 3).

517 As we previously mentioned, both S. uralensis and S. aluco exhibit a duplicated control

518 region structure in their mitochondrial genomes (Brito, 2005). Neither Omote et al. (2013) nor

519 Xiao et al. (2006) report the presence of a duplicated control region structure in either $S$.

520 uralensis or $S$. aluco, respectively, in their discussions of the repetitive content of the control

521 region sequences of these two species. It is not overtly clear from their methodologies which

522 control region they sequenced. The precise primer combinations used for the PCR-amplification

523 and sequencing of the control region of the Bubo species and S. uralensis are not provided by

524 Omote et al. (2013), but mapping the primer sequences used by the researchers to our S. $O$. 
525 caurina genome suggests that, if the structure of the $S$. uralensis mitochondrial genome shares

526 that of $S$. o. caurina, they likely sequenced CR2 in at least $S$. uralensis and in the Bubo species if

527 a CR2 was present. We are unsure how placement of the primers in $c y t b$ and $12 S$, as reported in

528 the methodology of Xiao et al. (2006) could PCR-amplify a single control region sequence for $S$.

529 aluco, given the duplicated control region structure (Brito, 2005).

530 The duplicated control region structure is unreported in Strigiformes outside of Strix, but

531 we infer that it is also likely present in Bubo due to the positioning of primers used for PCR-

532 amplification of the control region in previous studies (Marthinsen et al., 2009; Omote et al.,

533 2013). If also present in Bubo, then the duplicate control region structure appears to have arisen

534 in the common ancestor of Strix and Bubo, but a proper phylogenetic test of this hypothesis with

535 increased taxon sampling is warranted. Further work on the structure of control region sequences

536 in Strix and related taxa is needed to elucidate the pattern of evolution of this region across the

537 Strigidae phylogeny.

538 Although inconclusive and warranting further investigation, our evidence for two

539 versions of the 3' repetitive region of CR1 suggests that mitochondrial heteroplasmy is present in

540 this S. o. caurina individual. Mitochondrial heteroplasmy due to tandem repeat variability in the

541 control region has been shown to occur in other bird species (Berg, Moum \& Johansen, 1995;

542 Mundy, Winchell \& Woodruff, 1996). Previous work has suggested that the most likely

543 mechanism by which the gain and loss of such tandem repeat elements occurs in the

544 mitochondrial control region is that the repetitive region forms a stable, single-stranded

545 secondary structure and there is slippage during replication (Levinson \& Gutman, 1987;

546 Wilkinson \& Chapman, 1991; Fumagalli et al., 1996; Faber \& Stepien, 1998). Greater numbers

547 of repeats may improve the stability of the secondary structure (Faber \& Stepien, 1998). 
548 Utilizing sequence from the 3' region of CR1 for population genetic study of S. o. caurina is not

549 likely to be useful due to the variability (in terms of the number of copies of the tandem repeat

550 motifs in this region) that is potentially present within a single individual.

551 The 29,520 nt of Numt sequence in the draft S. o. caurina nuclear genome assembly is

552 more than triple the 8,869 nt of Numt sequence found in a Gallus gallus draft nuclear genome

553 assembly (Pereira \& Baker, 2004). The 3,280 nt average Numt size exceeds the average size in

554 all of the eukaryotic genomes examined by Richly \& Leister (2004). There are markedly fewer

555 control region Numts in the S. o. caurina draft genome assembly than found in a Gallus gallus

556 draft genome assembly (Pereira \& Baker, 2004). We only found one control region Numt (Table

557 4). Indeed the longest Numt, Numt \#1, extends through almost the entire mitochondrial genome

558 sequence including from $t R N A^{\text {Phe }}$ through $t R N A^{T h r}$, immediately adjacent to, but ending at CR1.

559 The percentage identity of the nuclear pseudogenes with the true mitochondrial genes ranges

560 from $77.5-87.81 \%$, so care must be taken to insure that Numts are not PCR-amplified in place of

561 mitochondrial gene sequences. As the control region is the mitochondrial segment that has been

562 used most often in studies of the population genetics of Strix species (Barrowclough, Gutierrez \&

563 Groth, 1999; Haig et al., 2004; Barrowclough et al., 2005; Brito, 2005; Hull et al., 2010;

564 Barrowclough et al., 2011), it is encouraging that only one, short Numt included CR1 or CR2

565 (Table 4). As long as researchers PCR-amplify sequences that span beyond the $592 \mathrm{nt}$ Numt \#9,

566 they should have confidence in amplifying the true mitochondrial control regions.

567 The average pairwise sequence divergence between S. occidentalis and S. varia has been

568 previously reported as $13.9 \%$ for a 524 nt section of CR1 (Haig et al., 2004). This exceeds the

569 weighted average of $10.74 \%(8.68 \%$ uncorrected $p$-distance $)$ that we calculated across the non-

570 tRNA mitochondrial genes (Table 5), which is unsurprising as the control region is known to be 
571 rapidly evolving in birds (Quinn \& Wilson, 1993). However, the pairwise sequence divergence

572 between $S$. occidentalis and S. varia appears higher in ND3, ND4L, ND6, and ATP8 (Table 5)

573 than in the CR1 portion. Hull et al. (2010) found uncorrected $p$-distances of $13.73-13.93 \%$ for

$574 N D 2$ and $14.58-14.81 \%$ for the control region between S. nebulosa and S. occidentalis. Wink \&

575 Heidrich (1999) calculated uncorrected $p$-distances of $8.15-11.72 \%$ for $c y t b$ sequence pairwise

576 comparisons of six Strix species (S. aluco, S. butleri, S. nebulosa, S. rufipes, S. uralensis, and S.

577 woodfordii). Of these six Strix species, only S. aluco x S. uralensis are known to hybridize

578 (McCarthy, 2006), albeit in captivity (Scherzinger, 1982), and their cyt b pairwise divergence

579 was $8.15 \%$, the lowest of those calculated by Wink \& Heidrich (1999) between Strix species.

580 Our $c y t b$ uncorrected $p$-distance value between $S$. o. caurina and $S$. varia was $9.21 \%$, which is

581 also on the lower end of the range of Wink \& Heidrich (1999) Strix interspecific divergences.

582 Overall, however, high levels of interspecific pairwise divergence of mitochondrial DNA seem

583 to be typical for the genus Strix, even for species able to hybridize. We anticipate that these

584 whole mitochondrial genome resources will be useful to those with an interest in developing new

585 mitochondrial markers to study the genetics of $S$. o. caurina, $S$. varia, and related taxa.

\section{Acknowledgements}

We thank WildCare, San Rafael for graciously providing us with blood samples from

Sequoia. We thank the Cincinnati Museum Center for providing a barred owl (Strix varia) tissue sample. We thank Laura Wilkinson for assistance with laboratory work. We generated genetic

591 sequence data at the Center for Comparative Genomics, California Academy of Sciences.

592 Genewiz, Inc. constructed and sequenced the Nextera (Illumina) large-insert mate-pair libraries.

593 The Genomics Core Facility at the Icahn Institute and Department of Genetics and Genomic 
594 Sciences, Icahn School of Medicine at Mount Sinai constructed and sequenced the Pacific

595 Biosciences library.

596

597 References

598 Altschul SF., Gish W., Miller W., Myers EW., Lipman DJ. 1990. Basic local alignment search tool. Journal of Molecular Biology 215:403-410. DOI: 10.1016/S0022-2836(05)80360-2.

600

Barrowclough GF., Groth JG., Mertz LA., Gutiérrez RJ. 2005. Genetic structure, introgression,

601

602

603

604

605

606

607

608

609

610

611

612

613

614

615

616 and a narrow hybrid zone between northern and California spotted owls (Strix occidentalis). Molecular Ecology 14:1109-1120. DOI: 10.1111/j.1365294X.2005.02465.x.

Barrowclough GF., Groth JG., Odom KJ., Lai JE. 2011. Phylogeography of the Barred Owl (Strix varia): Species Limits, Multiple Refugia, and Range Expansion. The Auk 128:696706. DOI: 10.1525/auk.2011.11057.

Barrowclough GF., Gutierrez RJ., Groth JG. 1999. Phylogeography of Spotted Owl (Strix occidentalis) Populations Based on Mitochondrial DNA Sequences: Gene Flow, Genetic Structure, and a Novel Biogeographic Pattern. Evolution 53:919-931. DOI: $10.2307 / 2640731$.

Bensch S., H A. 2000. Mitochondrial Genomic Rearrangements in Songbirds. Molecular Biology and Evolution 17:107-113.

Benson G. 1999. Tandem repeats finder: a program to analyze DNA sequences. Nucleic Acids Research 27:573-580. DOI: 10.1093/nar/27.2.573.

Benson G. 2012. Tandem Repeats Finder. Version 4.07b. [Accessed 2016 Oct 1]. Available from: https://tandem.bu.edu/trf/trf.html. 
617 Benson G. 2016. Tandem Repeats Finder. Version 4.09. [Accessed 2016 Oct 1]. Available from: $618 \quad$ https://tandem.bu.edu/trf/trf.html.

619 Benson DA., Clark K., Karsch-Mizrachi I., Lipman DJ., Ostell J., Sayers EW. 2015. GenBank. $620 \quad$ Nucleic Acids Research 43:D30-D35. DOI: 10.1093/nar/gku1216.

621 Berg T., Moum T., Johansen S. 1995. Variable numbers of simple tandem repeats make birds of 622 the order Ciconiiformes heteroplasmic in their mitochondrial genomes. Current Genetics 623 27:257-262. DOI: 10.1007/BF00326158.

624 Bernt M., Donath A., Jühling F., Externbrink F., Florentz C., Fritzsch G., Pütz J., Middendorf 625 M., Stadler PF. 2013. MITOS: Improved de novo metazoan mitochondrial genome annotation. Molecular Phylogenetics and Evolution 69:313-319. DOI: 10.1016/j.ympev.2012.08.023.

Biomatters 2016. Geneious. Version 9.1.4. [Accessed 2016 Oct 1]. Available from: http://www.geneious.com.

Bolger AM., Lohse M., Usadel B. 2014. Trimmomatic: a flexible trimmer for Illumina sequence data. Bioinformatics 30:2114-2120. DOI: 10.1093/bioinformatics/btu170.

632

633

634

635
Boratyn GM., Camacho C., Cooper PS., Coulouris G., Fong A., Ma N., Madden TL., Matten WT., McGinnis SD., Merezhuk Y., Raytselis Y., Sayers EW., Tao T., Ye J., Zaretskaya I. 2013. BLAST: a more efficient report with usability improvements. Nucleic Acids Research 41:W29-W33. DOI: 10.1093/nar/gkt282.

Brito PH. 2005. The influence of Pleistocene glacial refugia on tawny owl genetic diversity and phylogeography in western Europe. Molecular Ecology 14:3077-3094. DOI: 10.1111/j.1365-294X.2005.02663.x. 
639 Camacho C., Coulouris G., Avagyan V., Ma N., Papadopoulos J., Bealer K., Madden TL. 2009.

640 BLAST+: architecture and applications. BMC Bioinformatics 10:421. DOI:

641 $10.1186 / 1471-2105-10-421$.

642 Capella-Gutiérrez S., Gabaldón T. 2013. trimA1. Version 1.4.rev15. [Accessed 2016 Oct 1]. 643 Available from: https://github.com/scapella/trimal.

644 Capella-Gutiérrez S., Silla-Martínez JM., Gabaldón T. 2009. trimAl: a tool for automated 645 alignment trimming in large-scale phylogenetic analyses. Bioinformatics 25:1972-1973.

646 DOI: 10.1093/bioinformatics/btp348.

647

De Wit P., Pespeni MH., Ladner JT., Barshis DJ., Seneca F., Jaris H., Therkildsen NO., 648 Morikawa M., Palumbi SR. 2012. The simple fool's guide to population genomics via RNA-Seq: an introduction to high-throughput sequencing data analysis. Molecular Ecology Resources 12:1058-1067. DOI: 10.1111/1755-0998.12003.

Desjardins P., Morais R. 1990. Sequence and gene organization of the chicken mitochondrial genome. Journal of Molecular Biology 212:599-634. DOI: 10.1016/00222836(90)90225-B.

Eberhard JR., Wright TF. 2016. Rearrangement and evolution of mitochondrial genomes in 655 parrots. Molecular Phylogenetics and Evolution 94, Part A:34-46. DOI:

656 10.1016/j.ympev.2015.08.011.

657 Eberhard JR., Wright TF., Bermingham E. 2001. Duplication and Concerted Evolution of the 658 Mitochondrial Control Region in the Parrot Genus Amazona. Molecular Biology and Evolution 18:1330-1342.

660 Edgar RC. 2004. MUSCLE: multiple sequence alignment with high accuracy and high throughput. Nucleic Acids Research 32:1792-1797. DOI: 10.1093/nar/gkh340. 
662 Faber JE., Stepien CA. 1998. Tandemly Repeated Sequences in the Mitochondrial DNA Control 663 Region and Phylogeography of the Pike-Perches Stizostedion. Molecular Phylogenetics 664 and Evolution 10:310-322. DOI: 10.1006/mpev.1998.0530.

665 Free Software Foundation 2012. GNU Awk . Version 4.0.1. [Accessed 2016 Oct 1]. Available 666 from: https://www.gnu.org/software/gawk.

667 Free Software Foundation 2014. GNU Grep. Version 2.16. [Accessed 2016 Oct 1]. Available 668 from: https://www.gnu.org/software/grep.

669 Fuchs J., Pons J-M., Goodman SM., Bretagnolle V., Melo M., Bowie RC., Currie D., Safford R., Virani MZ., Thomsett S., Hija A., Cruaud C., Pasquet E. 2008. Tracing the colonization 10.1186/1471-2148-8-197.

674 Fumagalli L., Taberlet P., Favre L., Hausser J. 1996. Origin and evolution of homologous repeated sequences in the mitochondrial DNA control region of shrews. Molecular Biology and Evolution 13:31-46.

Funk WC., Mullins TD., Forsman ED., Haig SM. 2007. Microsatellite loci for distinguishing spotted owls (Strix occidentalis), barred owls (Strix varia), and their hybrids. Molecular Ecology Notes 7:284-286. DOI: 10.1111/j.1471-8286.2006.01581.x.

680 Gibb GC., Kardailsky O., Kimball RT., Braun EL., Penny D. 2007. Mitochondrial Genomes and 681 682 Avian Phylogeny: Complex Characters and Resolvability without Explosive Radiations. Molecular Biology and Evolution 24:269-280. DOI: 10.1093/molbev/ms1158. 
683 Guan X., Xu J., Smith EJ. 2016. The complete mitochondrial genome sequence of the 684 budgerigar, Melopsittacus undulatus. Mitochondrial DNA Part A 27:401-402. DOI: 685 $10.3109 / 19401736.2014 .898277$.

686 687 688 689

Haddrath O., Baker AJ. 2001. Complete mitochondrial DNA geonome sequences of extinct birds: ratite phylogenetics and the vicariance biogeography hypothesis. Proceedings of the Royal Society of London B: Biological Sciences 268:939-945. DOI: 10.1098/rspb.2001.1587.

Haig SM., Mullins TD., Forsman ED., Trail PW., Wennerberg L. 2004. Genetic identification of spotted owls, barred owls, and their hybrids: legal implications of hybrid identity. Conservation Biology 18:1347-1357.

Hanna ZR., Henderson JB., Wall JD., Emerling CA., Fuchs J., Runckel C., Mindell DP., Bowie RCK., DeRisi JL., Dumbacher JP. 2017. Northern spotted owl (Strix occidentalis caurina) genome: divergence with the barred owl (Strix varia) and characterization of light-associated genes. Genome Biology and Evolution evx158. DOI: 10.1093/gbe/evx 158 .

Haring E., Kruckenhauser L., Gamauf A., Riesing MJ., Pinsker W. 2001. The Complete Sequence of the Mitochondrial Genome of Buteo buteo (Aves, Accipitridae) Indicates an Early Split in the Phylogeny of Raptors. Molecular Biology and Evolution 18:1892-1904.

Harrison GL (Abby)., McLenachan PA., Phillips MJ., Slack KE., Cooper A., Penny D. 2004. Four New Avian Mitochondrial Genomes Help Get to Basic Evolutionary Questions in the Late Cretaceous. Molecular Biology and Evolution 21:974-983. DOI: 10.1093/molbev/msh065. 
705 Hausknecht R., Jacobs S., Müller J., Zink R., Frey H., Solheim R., Vrezec A., Kristin A., Mihok

706 J., Kergalve I., Saurola P., Kuehn R. 2014. Phylogeographic analysis and genetic cluster

707 recognition for the conservation of Ural Owls (Strix uralensis) in Europe. Journal of

708 Ornithology 155:121-134. DOI: 10.1007/s10336-013-0994-8.

709 Henderson JB., Hanna ZR. 2016a. BLATq. Version 1.0.2. Zenodo. DOI: 10.5281/zenodo.61136.

710 Henderson JB., Hanna ZR. 2016b. excerptByIDs. Version 1.0.2. Zenodo. DOI:

711 $10.5281 /$ zenodo.61134.

712 Hengjiu T., Jianwei J., Shi Y., Zhiming Z., Laghari MY., Narejo NT., Lashari P. 2016. Complete mitochondrial genome of Eagle Owl (Bubo bubo, Strigiformes; Strigidae) from China. Mitochondrial DNA Part A 27:1455-1456. DOI: 10.3109/19401736.2014.953090.

Hull JM., Englis A., Medley JR., Jepsen EP., Duncan JR., Ernest HB., Keane JJ. 2014. A New Subspecies of Great Gray Owl (Strix nebulosa) in the Sierra Nevada of California, U.S.A. Journal of Raptor Research 48:68-77. DOI: 10.3356/JRR-13-35.1.

Hull JM., Keane JJ., Savage WK., Godwin SA., Shafer JA., Jepsen EP., Gerhardt R., Stermer C., Ernest HB. 2010. Range-wide genetic differentiation among North American great gray owls (Strix nebulosa) reveals a distinct lineage restricted to the Sierra Nevada, California. Molecular Phylogenetics and Evolution 56:212-221. DOI: 10.1016/j.ympev.2010.02.027.

Johnson M., Zaretskaya I., Raytselis Y., Merezhuk Y., McGinnis S., Madden TL. 2008. NCBI BLAST: a better web interface. Nucleic Acids Research 36:W5-W9. DOI: 10.1093/nar/gkn201.

Katoh K. 2016. MAFFT: a multiple sequence alignment program. Version 7.305b. [Accessed 2016 Oct 1]. Available from: http://mafft.cbrc.jp/alignment/software. 
728 Katoh K., Misawa K., Kuma K., Miyata T. 2002. MAFFT: a novel method for rapid multiple 729 sequence alignment based on fast Fourier transform. Nucleic Acids Research 30:3059-

730 3066. DOI: 10.1093/nar/gkf436.

731 Katoh K., Standley DM. 2013. MAFFT Multiple Sequence Alignment Software Version 7:

732 Improvements in Performance and Usability. Molecular Biology and Evolution 30:772-

733 780. DOI: $10.1093 / \mathrm{molbev} / \mathrm{mst} 010$.

734 Kearse M., Moir R., Wilson A., Stones-Havas S., Cheung M., Sturrock S., Buxton S., Cooper A.,

735 Markowitz S., Duran C., Thierer T., Ashton B., Meintjes P., Drummond A. 2012.

736 Geneious Basic: An integrated and extendable desktop software platform for the organization and analysis of sequence data. Bioinformatics 28:1647-1649. DOI: 10.1093/bioinformatics/bts199.

Kelly EG., Forsman ED. 2004. Recent Records of Hybridization Between Barred Owls (Strix varia) and Northern Spotted Owls (S. occidentalis caurina). The Auk 121:806-810. DOI:

Kent WJ. 2002. BLAT—The BLAST-Like Alignment Tool. Genome Research 12:656-664. DOI: $10.1101 /$ gr.229202.

744 Kent WJ. 2012. BLAT-The BLAST-Like Alignment Tool. Version 35. [Accessed 2016 Oct 1]. Available from: https://users.soe.ucsc.edu/ kent.

Krosby M., Rohwer S. 2009. A 2000 Km Genetic Wake Yields Evidence for Northern Glacial 
749 Kumar S., Stecher G., Tamura K. 2016. MEGA7: Molecular Evolutionary Genetics Analysis

750 version 7.0 for bigger datasets. Molecular Biology and Evolution 33:1870-1874. DOI:

$751 \quad 10.1093 / \mathrm{molbev} / \mathrm{msw} 054$.

752 Kumar S., Tamura K., Nei M. 1994. MEGA: Molecular Evolutionary Genetics Analysis software 753 for microcomputers. Computer applications in the biosciences : CABIOS 10:189-191.

754 DOI: 10.1093/bioinformatics/10.2.189.

755 Kumazawa Y., Ota H., Nishida M., Ozawa T. 1996. Gene rearrangements in snake mitochondrial genomes: highly concerted evolution of control-region-like sequences duplicated and inserted into a tRNA gene cluster. Molecular Biology and Evolution 13:1242-1254. DOI: 10.1093/oxfordjournals.molbev.a025690.

759 760

761 762
Levinson G., Gutman GA. 1987. Slipped-strand mispairing: a major mechanism for DNA sequence evolution. Molecular Biology and Evolution 4:203-221.

Li H. 2013a. Aligning sequence reads, clone sequences and assembly contigs with BWA-MEM. arXiv:1303.3997 [q-bio].

Li H. 2013b. bioawk. Version 1.0. [Accessed 2016 Oct 1]. Available from: https://github.com/lh3/bioawk.

Li H., Handsaker B., Marshall J., Danecek P. 2015. Samtools. Version 1.3 with HTSlib 1.3.1. [Accessed 2016 Oct 1]. Available from: http://www.htslib.org.

Li H., Handsaker B., Wysoker A., Fennell T., Ruan J., Homer N., Marth G., Abecasis G., Durbin R., Subgroup 1000 Genome Project Data Processing. 2009. The Sequence Alignment/Map format and SAMtools. Bioinformatics 25:2078-2079. DOI: 10.1093/bioinformatics/btp352. 
771 Liu G., Zhou L., Gu C. 2014. The complete mitochondrial genome of Brown wood owl Strix leptogrammica (Strigiformes: Strigidae). Mitochondrial DNA 25:370-371. DOI:

773 10.3109/19401736.2013.803540.

774

775

776

777

778

779

780

781

782

783

784

785

786

787

788

789

790

791

792

793

Lopez JV., Yuhki N., Masuda R., Modi W., O’Brien SJ. 1994. Numt, a recent transfer and tandem amplification of mitochondrial DNA to the nuclear genome of the domestic cat. Journal of Molecular Evolution 39:174-190.

Luo R., Liu B., Xie Y., Li Z., Huang W., Yuan J., He G., Chen Y., Pan Q., Liu Y., Tang J., Wu G., Zhang H., Shi Y., Liu Y., Yu C., Wang B., Lu Y., Han C., Cheung DW., Yiu S-M., Peng S., Xiaoqian Z., Liu G., Liao X., Li Y., Yang H., Wang J., Lam T-W., Wang J. 2012. SOAPdenovo2: an empirically improved memory-efficient short-read de novo assembler. GigaScience 1:18. DOI: 10.1186/2047-217X-1-18.

Marshall HD., Baker AJ. 1997. Structural conservation and variation in the mitochondrial control region of fringilline finches (Fringilla spp.) and the greenfinch (Carduelis chloris). Molecular Biology and Evolution 14:173-184.

Marthinsen G., Wennerberg L., Solheim R., Lifjeld JT. 2009. No phylogeographic structure in the circumpolar snowy owl (Bubo scandiacus). Conservation Genetics 10:923-933. DOI: $10.1007 / \mathrm{s} 10592-008-9581-6$.

Mazur KM., James PC. 2000. Barred Owl (Strix varia). The Birds of North America Online (A. Poole, Ed.) Ithaca: Cornell Lab of Ornithology. [Accessed 2016 Oct 1]. Retrieved from the Birds of North America Online: https://birdsna.org/SpeciesAccount/bna/species/brdowl. DOI: 10.2173/bna.508.

McCarthy EM. 2006. Handbook of avian hybrids of the world. New York, New York: Oxford University Press. 
794 Mindell DP., Sorenson MD., Dimcheff DE. 1998a. Multiple independent origins of

795 mitochondrial gene order in birds. Proceedings of the National Academy of Sciences

$796 \quad 95: 10693-10697$.

797 Mindell DP., Sorenson MD., Dimcheff DE. 1998b. An extra nucleotide is not translated in 798 mitochondrial ND3 of some birds and turtles. Molecular Biology and Evolution 15:1568$799 \quad 1571$.

800 Mindell DP., Sorenson MD., Dimcheff DE., Hasegawa M., Ast JC., Yuri T. 1999. Interordinal

801 Relationships of Birds and Other Reptiles Based on Whole Mitochondrial Genomes.

$802 \quad$ Systematic Biology 48:138-152. DOI: 10.1080/106351599260490.

803 Mindell DP., Sorenson MD., Huddleston CJ., Miranda HC., Knight A., Sawchuk SJ., Yuri T.

804 1997. Phylogenetic Relationships among and within Select Avian Orders Based on

805 Mitochondrial DNA. In: Avian Molecular Evolution and Systematics. San Diego,

$806 \quad$ California: Academic Press, 211-245.

807 Morgulis A., Coulouris G., Raytselis Y., Madden TL., Agarwala R., Schäffer AA. 2008.

808 Database indexing for production MegaBLAST searches. Bioinformatics 24:1757-1764.

809 DOI: $10.1093 /$ bioinformatics/btn322.

810 Mundy NI., Winchell CS., Woodruff DS. 1996. Tandem Repeats and Heteroplasmy in the

811 Mitochondrial DNA Control Region of the Loggerhead Shrike (Lanius ludovicianus).

$812 \quad$ Journal of Heredity 87:21-26.

813 NCBI Resource Coordinators 2015. Database resources of the National Center for Biotechnology

814 Information. Nucleic Acids Research 43:D6-D17. DOI: 10.1093/nar/gku1130.

815 Omote K., Nishida C., Dick MH., Masuda R. 2013. Limited phylogenetic distribution of a long 816 tandem-repeat cluster in the mitochondrial control region in Bubo (Aves, Strigidae) and 
cluster variation in Blakiston's fish owl (Bubo blakistoni). Molecular Phylogenetics and

818 Evolution 66:889-897. DOI: 10.1016/j.ympev.2012.11.015.

819 Pereira SL., Baker AJ. 2004. Low number of mitochondrial pseudogenes in the chicken (Gallus 820 gallus) nuclear genome: implications for molecular inference of population history and 821 phylogenetics. BMC Evolutionary Biology 4:17. DOI: 10.1186/1471-2148-4-17.

822 Pratt RC., Gibb GC., Morgan-Richards M., Phillips MJ., Hendy MD., Penny D. 2009. Toward 823 Resolving Deep Neoaves Phylogeny: Data, Signal Enhancement, and Priors. Molecular Biology and Evolution 26:313-326. DOI: 10.1093/molbev/msn248.

825 Quinlan AR., Hall IM. 2010. BEDTools: a flexible suite of utilities for comparing genomic 826 features. Bioinformatics 26:841-842. DOI: 10.1093/bioinformatics/btq033.

827 Quinn TW., Wilson AC. 1993. Sequence evolution in and around the mitochondrial control 828 region in birds. Journal of Molecular Evolution 37:417-425. DOI: 10.1007/BF00178871.

829 Randi E., Lucchini V. 1998. Organization and evolution of the mitochondrial DNA control 830 region in the avian genus Alectoris. Journal of Molecular Evolution 47:449-462.

Richly E., Leister D. 2004. NUMTs in Sequenced Eukaryotic Genomes. Molecular Biology and Evolution 21:1081-1084. DOI: 10.1093/molbev/msh110.

833 Ruby JG. 2014. PRICE. Version 1.2. [Accessed 2016 Oct 1]. Available from:

834 http://derisilab.ucsf.edu/software/price.

835 Ruby JG., Bellare P., DeRisi JL. 2013. PRICE: Software for the Targeted Assembly of 836 Components of (Meta) Genomic Sequence Data. G3: Genes|Genomes|Genetics 3:865837 880. DOI: $10.1534 / \mathrm{g} 3.113 .005967$. 
838 Ruegg K. 2008. Genetic, Morphological, and Ecological Characterization of a Hybrid Zone That 839 Spans a Migratory Divide. Evolution 62:452-466. DOI: 10.1111/j.1558$840 \quad$ 5646.2007.00263.x.

841 Scherzinger W. 1982. Beobachtungen an WaldkauzHabichtskauz-Hybriden (Strix aluco $\times$ Strix 842 uralensis). Zoologische Gärten 53:133-148.

843 Sorenson MD., Quinn TW. 1998. Numts: A Challenge for Avian Systematics and Population 844 Biology. The Auk 115:214-221. DOI: 10.2307/4089130.

845 Sun X., Zhou W., Sun Z., Qian L., Zhang Y., Pan T., Zhang B. 2016. The complete 846 mitochondrial genome of Glaucidium brodiei (Strigiformes: Strigidae). Mitochondrial 847 DNA Part A 27:2508-2509. DOI: 10.3109/19401736.2015.1036252.

848 Tamura K., Nei M. 1993. Estimation of the number of nucleotide substitutions in the control 849 region of mitochondrial DNA in humans and chimpanzees. Molecular Biology and Evolution 10:512-526.

851 Wada Y., Yamada Y., Nishibori M., Yasue H. 2004. Complete Nucleotide Sequence of 852 853 Mitochondrial Genome in Silkie Fowl (Gallus gallus var. domesticus). The Journal of Poultry Science 41:76-82. DOI: 10.2141/jpsa.41.76.

854 Wilkinson GS., Chapman AM. 1991. Length and sequence variation in evening bat D-loop 855 mtDNA. Genetics 128:607-617.

856 Williford D., DeYoung RW., Honeycutt RL., Brennan LA., Hernández F., Heffelfinger JR., 857 Harveson LA. 2014. Phylogeography of the Gambel's Quail (Callipepla gambelii) of 858 western North America. The Wilson Journal of Ornithology 126:218-235. DOI:

859 10.1676/13-111.1. 
860 Wink M., El-Sayed A-A., Sauer-Gürth H., Gonzalez J. 2009. Molecular Phylogeny of Owls

861 (Strigiformes) Inferred from DNA Sequences of the Mitochondrial Cytochrome $b$ and the 862 Nuclear RAG-1 gene. Ardea 97:581-591. DOI: 10.5253/078.097.0425.

863 Wink M., Heidrich P. 1999. Molecular Evolution and Systematics of the Owls (Strigiformes). In: 864 Owls - A Guide to the Owls of the World. Sussex, United Kingdom: Pica Press, 39-57.

865 Xiao B., Ma F., Sun Y., Li Q-W. 2006. Comparative Analysis of Complete Mitochondrial DNA 866 Control Region of Four Species of Strigiformes. Acta Genetica Sinica 33:965-974. DOI:

867 $10.1016 / \mathrm{S} 0379-4172(06) 60131-5$.

868 Zhang Z., Schwartz S., Wagner L., Miller W. 2000. A Greedy Algorithm for Aligning DNA 869 Sequences. Journal of Computational Biology 7:203-214. DOI:

870 $10.1089 / 10665270050081478$.

871 Zhang Y., Song T., Pan T., Sun X., Sun Z., Qian L., Zhang B. 2016. Complete sequence and 872 gene organization of the mitochondrial genome of Asio flammeus (Strigiformes, 873 strigidae). Mitochondrial DNA Part A 27:2665-2667. DOI: 10.3109/19401736.2015.1043538.

875 Zink RM. 1994. The Geography of Mitochondrial DNA Variation, Population Structure, 876 Hybridization, and Species Limits in the Fox Sparrow (Passerella iliaca). Evolution 877 48:96-111. DOI: $10.2307 / 2410006$.

878

879 


\section{Table $\mathbf{1}$ (on next page)}

Strix specimen data.

We here provide further information regarding the datasets that archive the Strix specimens to which we refer throughout the manuscript. 


\begin{tabular}{|c|l|l|l|}
\hline \multicolumn{1}{|c|}{ Specimen } & \multicolumn{1}{c|}{ Data publisher } & \multicolumn{1}{c|}{$\begin{array}{c}\text { Date } \\
\text { accessed }\end{array}$} & \multicolumn{1}{c|}{ Link to dataset } \\
\hline CAS:ORN:95964 & $\begin{array}{l}\text { CAS Ornithology (ORN), California Academy of } \\
\text { Sciences, San Francisco, California, United States } \\
\text { of America }\end{array}$ & 2016 Aug 15 & $\begin{array}{l}\text { http://ipt.calacademy.org:8080/ipt/resour } \\
\text { ce.do?r=orn }\end{array}$ \\
\hline CAS:ORN:98821 & $\begin{array}{l}\text { CAS Ornithology (ORN), California Academy of } \\
\text { Sciences, San Francisco, California, United States } \\
\text { of America }\end{array}$ & 2016 Aug 15 & $\begin{array}{l}\text { http://ipt.calacademy.org:8080/ipt/resour } \\
\text { ce.do?r=orn }\end{array}$ \\
\hline CMC:ORNI-T:B41533 & $\begin{array}{l}\text { CMC ORNI-T, Museum of Natural History \& } \\
\text { Science, Cincinnati Museum Center, Cincinnati, } \\
\text { Ohio, United States of America }\end{array}$ & $\begin{array}{l}\text { 2017 Sep 3 } \\
\text { https://www.idigbio.org/portal/records/5 } \\
7 \mathrm{~d} 299 \mathrm{f} 0-2 \mathrm{cc} 7-44 \mathrm{f} 7 \text {-aa5f-3c2ea175e757 }\end{array}$ \\
\hline
\end{tabular}

1 


\section{Table 2 (on next page)}

Sequence of primers used in Sanger sequencing of control regions.

These are the sequences of all of the primers that we used to amplify control regions 1 and 2 in order to confirm the final sequence of these regions in the mitochondrial genome assemblies. 


\begin{tabular}{|c|c|c|c|c|c|}
\hline Primer name & $\begin{array}{c}\text { Relevant } \\
\text { region }\end{array}$ & Species used on & \begin{tabular}{|c|} 
External \\
or \\
Internal
\end{tabular} & Primer sequence $\left(5^{\prime} \rightarrow 3^{\prime}\right)$ & Source \\
\hline cytb-F1 & CR1 & S. o. caurina, S. varia & External & ATCCTCATTCTCTTCCCCGT & This study \\
\hline $17122 \mathrm{R}$ & CR1 & S. o. caurina, S. varia & External & GGTGGGGGTTATTATTAACTTT & This study \\
\hline CR1-F1 & CR1 & S. o. caurina, S. varia & Internal & CTCSASCAAATCCCAAGTTT & This study \\
\hline CR1-F1-RC & CR1 & S. o. caurina, S. varia & Internal & AAACTTGGGATTTGSTSGAG & This study \\
\hline CR1-R2 & CR1 & S. o. caurina, S. varia & Internal & GGAGGGCGAGAATAGTTGRT & This study \\
\hline CR1-R2-RC & CR1 & S. o. caurina, S. varia & Internal & AYCAACTATTCTCGCCCTCC & This study \\
\hline N1 & CR1 & S. o. caurina & Internal & AACATTGGTCTTGTAAACCAA & Barrowclough et al. 1999 \\
\hline $41 \mathrm{R}$ & CR2 & S. o. caurina & External & GCATCTTCAGTGCCATGCTT & This study \\
\hline $17572 \mathrm{~F}$ & CR2 & S. o. caurina & External & ATTATCCAAGGTCTGCGGCC & This study \\
\hline $17589 \mathrm{~F}$ & CR2 & S. o. caurina & Internal & GCCTGAAAAACCGCCGTTAA & This study \\
\hline $18327 \mathrm{~F}$ & CR2 & S. o. caurina & Internal & CACTTTTGCGCCTCTGGTTC & This study \\
\hline 19911R & CR2 & S. o. caurina & Internal & AGAGAGGCTCTGATTGCTTG & This study \\
\hline ND6-ext1F & CR2 & S. varia & External & ACAACCCCATAATAYGGCGA & This study \\
\hline $12 \mathrm{~S}-\mathrm{ext1R}$ & CR2 & S. varia & External & GGTAGATGGGCATTTACACT & This study \\
\hline final-CR2F & CR2 & S. varia & Internal & TCAAACCAAACGATCGAGAA & This study \\
\hline $18547 \mathrm{~F}$ & CR2 & S. varia & Internal & CTCACGTGAAATCAGCAACC & This study \\
\hline 19088R & CR2 & S. varia & Internal & ATTCAACTAAAATTCGTTACAAATCTT & This study \\
\hline 19088R-RC & CR2 & S. varia & Internal & AAGATTTGTAACGAATTTTAGTTGAAT & This study \\
\hline
\end{tabular}




\section{Table 3 (on next page)}

Tandem repeat annotations.

This summarizes the repetitive regions of the northern spotted owl (Strix occidentalis caurina

or S. o. caurina) and barred owl (S. varia) mitochondrial genomes annotated by Tandem Repeats Finder. "Period size" refers to the size of the repeated motif. "Copy number" refers to the number of copies of the repeat in the region. "Consensus size" is the length of the consensus sequence summarizing all copies of the repeat, which may or may not be different from the period size. "Percent matches" refers to the percentage of nucleotides that match between adjacent copies of the repeat. "Percent indels" refers to the percentage of indels between adjacent copies of the repeat. We present the percent composition of each of the four nucleotides in the repetitive region. We have included the genomic regions that intersect each repetitive span in the "Region" column. "CR1" and "CR2" refer to control region 1 and control region 2, respectively. 


\begin{tabular}{|c|c|c|c|c|c|c|c|c|c|c|c|}
\hline Taxon & Coordinates (nt) & Region & \begin{tabular}{|c|} 
Period \\
Size \\
$(n t)$
\end{tabular} & $\begin{array}{c}\text { Copy } \\
\text { Number }\end{array}$ & $\begin{array}{c}\text { Consensus } \\
\text { Size (nt) }\end{array}$ & $\begin{array}{c}\text { Percent } \\
\text { Matches } \\
(\%)\end{array}$ & $\begin{array}{c}\text { Percent } \\
\text { Indels } \\
(\%)\end{array}$ & $\begin{array}{c}\mathrm{A} \\
(\%)\end{array}$ & $\begin{array}{c}\mathrm{C} \\
(\%)\end{array}$ & $\begin{array}{c}G \\
(\%)\end{array}$ & $\begin{array}{c}\mathrm{T} \\
(\%)\end{array}$ \\
\hline S. o. caurina & $10,267-10,309$ & ND4 & 18 & 2.3 & 19 & 84 & 4 & 25 & 46 & 0 & 27 \\
\hline S. o. caurina & $15,066-15,162$ & CR1 & 22 & 4.3 & 22 & 70 & 7 & 37 & 27 & 6 & 28 \\
\hline S. o. caurina & $15,169-15,311$ & CR1 & 67 & 2.1 & 67 & 83 & 8 & 40 & 31 & 6 & 21 \\
\hline S. o. caurina & $16,243-16,715$ & CR1 & 70 & 6.8 & 70 & 98 & 1 & 39 & 21 & 4 & 33 \\
\hline S. o. caurina & $16,245-16,715$ & CR1 & 139 & 3.4 & 139 & 99 & 0 & 39 & 22 & 4 & 33 \\
\hline S. o. caurina & $16,403-16,515$ & CR1 & 37 & 3.2 & 37 & 61 & 27 & 40 & 23 & 3 & 32 \\
\hline S. o. caurina & $17,679-17,795$ & CR2 & 44 & 2.6 & 45 & 87 & 4 & 43 & 30 & 4 & 21 \\
\hline S. o. caurina & $17,719-17,795$ & CR2 & 22 & 3.5 & 22 & 89 & 0 & 45 & 31 & 3 & 19 \\
\hline S. o. caurina & $18,798-19,076$ & CR2 & 70 & 4.0 & 70 & 99 & 0 & 39 & 21 & 4 & 34 \\
\hline S. o. caurina & $18,800-19,076$ & CR2 & 139 & 2.0 & 139 & 100 & 0 & 39 & 21 & 4 & 34 \\
\hline S. o. caurina & $18,958-19,070$ & CR2 & 37 & 3.2 & 37 & 61 & 27 & 40 & 23 & 3 & 32 \\
\hline S. o. caurina & $19,110-19,853$ & CR2 & 78 & 9.5 & 78 & 99 & 0 & 41 & 15 & 15 & 27 \\
\hline S. varia & $15,126-15,209$ & CR1 & 22 & 3.8 & 22 & 82 & 4 & 36 & 27 & 4 & 30 \\
\hline S. varia & $15,193-15,340$ & CR1 & 67 & 2.2 & 68 & 83 & 1 & 37 & 32 & 8 & 22 \\
\hline S. varia & $17,384-17,482$ & CR2 & 22 & 4.4 & 23 & 87 & 5 & 41 & 34 & 5 & 19 \\
\hline S. varia & $18,548-18,951$ & CR2 & 78 & 5.2 & 77 & 93 & 2 & 40 & 17 & 15 & 26 \\
\hline
\end{tabular}




\section{Table 4 (on next page)}

Mitochondrion-derived nuclear pseudogenes (Numts) identified in the Strix occidentalis caurina nuclear genome sequence and statistics of the results of BLASTN searches.

We indicate the mitochondrial genes that a Numt spans in the "Genes included" column. If a Numt spans more than two genes, we indicate the first and last genes that it spans as well as a gene in the middle of the Numt in order to indicate the direction that the Numt extends. The Numt additionally spans all of the intervening genes in such cases. "Start mtDNA" and "End mtDNA" indicate the mitochondrial genome assembly sequence positions and "Start Scaffold" and "End Scaffold" denote the nuclear genome assembly contig/scaffold sequence positions in the alignments of the mitochondrial genome assembly to the nuclear genome assembly. "\% ID" indicates the percentage of identical matches in an alignment. "E-value" is the Expect value. "Bit score" is a log-scaled version of the alignment score. We characterized some of the Numts by examining more than one alignment and concluding that a Numt spanned across those individual alignments. 


\begin{tabular}{|c|c|c|c|c|c|c|c|c|c|c|c|c|}
\hline $\begin{array}{c}\text { Numt } \\
\#\end{array}$ & Genes included & $\begin{array}{c}\text { Start } \\
\text { mtDNA }\end{array}$ & $\begin{array}{c}\text { End } \\
\text { mtDNA }\end{array}$ & $\begin{array}{l}\text { Nuclear } \\
\text { Genome } \\
\text { Scaffold } \\
\end{array}$ & $\begin{array}{c}\text { Start } \\
\text { Scaffold }\end{array}$ & $\begin{array}{c}\text { End } \\
\text { Scaffold }\end{array}$ & $\begin{array}{l}\text { Orien- } \\
\text { tation }\end{array}$ & $\%$ ID & E-value & \begin{tabular}{|c|} 
Bit \\
score \\
\end{tabular} & \begin{tabular}{|c|} 
Length \\
alignment \\
(nt)
\end{tabular} & $\begin{array}{c}\text { Length } \\
\text { Numt } \\
\text { (nt) }\end{array}$ \\
\hline & $t R N A^{\text {Phe }}-12 S-16 \mathrm{~S}$ & & 2,225 & scaffold478 & 47,666 & 49,858 & + & 79.92 & 0.0 & 1,565 & 2,261 & 19,522 \\
\hline & $16 S$ & 2,367 & 2,645 & scaffold478 & 49,871 & 50,143 & + & 87.81 & $2.16 \mathrm{e}-84$ & 322 & 279 & \\
\hline & $16 S-N D 2-t R N A^{A s n}$ & 2,706 & 5,223 & scaffold 478 & 50,161 & 52,680 & + & 80.66 & 0.0 & 1,921 & 2,549 & \\
\hline & $t R N A^{A s n}-C O I-t R N A^{S e r} 2$ & 5,219 & 6,932 & scaffold478 & 57,635 & 59,328 & + & 83.22 & 0.0 & 1,552 & 1,716 & \\
\hline & $t R N A^{S e r} 2-t R N A^{A s p}-C O I I$ & 6,988 & 7,103 & scaffold478 & 59,382 & 59,496 & + & 87.18 & $1.41 \mathrm{e}-26$ & 130 & 117 & \\
\hline & ATP6 - ND4 - ND5 & 8,382 & 13,249 & scaffold478 & 59,498 & 64,306 & + & 80.59 & 0.0 & 3,672 & 4,893 & \\
\hline & cyt $b$ & 14,047 & 14,733 & scaffold478 & 44,785 & 45,459 & + & 82.82 & $1.92 \mathrm{e}-169$ & 604 & 687 & \\
\hline & $t R N A^{T h r}$ & 14,729 & 14,878 & scaffold478 & 46,066 & 46,222 & + & 82.80 & $1.09 \mathrm{e}-27$ & 134 & 157 & \\
\hline & $16 S$ & 1,682 & 2,603 & scaffold215 & $5,517,239$ & $5,518,161$ & - & 81.97 & 0.0 & 773 & 932 & 923 \\
\hline & $t R N A^{\mathrm{Ser} 2}-A T P 8-N D 3 \quad a$ & 6,989 & 9,584 & scaffold215 & $5,513,222$ & $5,515,749$ & - & 79.01 & 0.0 & 1,690 & 2615 & 2,528 \\
\hline & $16 S-t R N A^{\text {Leu } 2}$ & 2,290 & 2,788 & scaffold632 & $1,548,886$ & $1,549,372$ & + & 77.50 & $6.14 \mathrm{e}-70$ & 274 & 511 & 487 \\
\hline & $N D 1-t R N A^{G l n}-N D 2$ & 2,810 & 4,646 & scaffold167 & $11,322,764$ & $11,324,590$ & + & 80.54 & \begin{tabular}{|r|}
0.0 \\
\end{tabular} & 1,400 & 1,840 & 2,732 \\
\hline & $N D 2-t R N A^{A s n}-C O I$ & 4,692 & 5,597 & scaffold167 & $11,324,598$ & $11,325,495$ & + & 83.68 & 0.0 & 846 & 907 & \\
\hline & $t R N A^{G l u}-N D 2-C O I$ & 3,851 & 5,526 & scaffold 1500 & 35,914 & 37,582 & - & 84.21 & 0.0 & 1,620 & 1,678 & 1,669 \\
\hline & $N D 2-t R N A^{A s n}-t R N A^{T y r}$ & 4,500 & 5,348 & scaffold173 & 750,945 & 751,785 & - & 81.40 & 0.0 & 680 & 855 & 841 \\
\hline & ND5 & 12,082 & 12,310 & scaffold143 & 586,822 & 587,047 & + & 81.30 & $3.83 e-42$ & 182 & 230 & 226 \\
\hline & $C R 1$ & 15,026 & 15,640 & scaffold294 & $2,356,468$ & $2,357,059$ & - & 83.07 & $9.17 \mathrm{e}-148$ & 532 & 620 & 592 \\
\hline & CR2 & 17,677 & 18,195 & scaffold294 & $2,356,468$ & $2,356,986$ & - & 80.87 & $9.70 \mathrm{e}-108$ & 399 & 528 & \\
\hline
\end{tabular}




\section{Table 5 (on next page)}

Divergence of Strix occidentalis caurina and Strix varia at all protein-coding genes.

This provides the number of base substitutions per site for all mitochondrial protein-coding genes and rRNAs between the mitochondrial sequences of Strix occidentalis occidentalis and S. varia. P-distance refers to an uncorrected pairwise distance while TN93 refers to the pairwise distance corrected by the Tamura-Nei 1993 model (Tamura et al., 1993). 


\begin{tabular}{|l|r|r|r|}
\hline Gene & $\begin{array}{c}\text { Number of } \\
\text { sites in } \\
\text { alignment (nt) }\end{array}$ & p-distance & $\begin{array}{c}\text { Distance } \\
\text { with TN93 } \\
\text { model }\end{array}$ \\
\hline $12 S$ & 984 & $5.79 \%$ & $6.61 \%$ \\
\hline $16 S$ & 1,589 & $5.48 \%$ & $6.14 \%$ \\
\hline ATP6 & 681 & $9.10 \%$ & $11.07 \%$ \\
\hline ATP8 & 165 & $14.55 \%$ & $20.81 \%$ \\
\hline COI & 1,548 & $7.88 \%$ & $9.31 \%$ \\
\hline COII & 681 & $9.10 \%$ & $11.23 \%$ \\
\hline COII & 783 & $7.54 \%$ & $8.89 \%$ \\
\hline cyt_b & 1,140 & $9.21 \%$ & $11.35 \%$ \\
\hline ND1 & 957 & $10.66 \%$ & $13.46 \%$ \\
\hline ND2 & 1,038 & $9.34 \%$ & $11.60 \%$ \\
\hline ND3_a & 174 & $10.92 \%$ & $13.96 \%$ \\
\hline ND3_b & 174 & $11.49 \%$ & $14.86 \%$ \\
\hline ND4 & 1,377 & $10.31 \%$ & $13.12 \%$ \\
\hline ND4L & 294 & $11.22 \%$ & $14.29 \%$ \\
\hline ND5 & 1,818 & $9.19 \%$ & $11.29 \%$ \\
\hline ND6 & 516 & $9.69 \%$ & $14.71 \%$ \\
\hline
\end{tabular}

1 


\section{Figure 1}

Ancestral avian mitochondrial gene order surrounding the control region compared with that of Strix occidentalis caurina and Strix varia.

The Chicken panel displays the gene order of Gallus gallus, which is the presumed ancestral avian gene order. The Spotted Owl panel depicts the gene order of Strix occidentalis caurina and the Barred Owl panel depicts the gene order of Strix varia. All rRNAs, tRNAs, and proteincoding genes outside of the displayed region exhibit the same order in all of these mitochondrial genomes. "CR" denotes the control region with "CR1" and "CR2" referring to control regions 1 and 2, respectively. We added 100 nucleotides to each of the tRNAs to improve visualization. Apart from the tRNAs, the annotations are to scale relative to each other with the numbers at the top of the figure denoting nucleotides. The order of the genes outside of the region depicted in this figure is the same in the chicken, spotted owl, and barred owl.

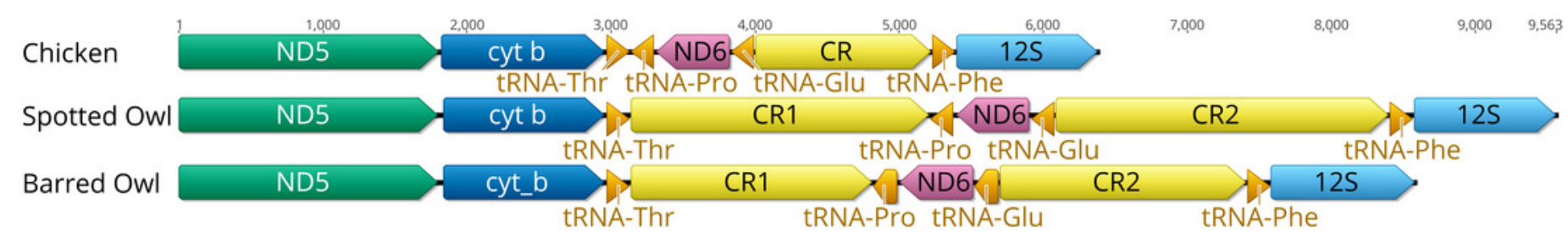




\section{Figure 2}

Alignment of control regions 1 and 2 within Strix occidentalis caurina and Strix varia.

Panel A depicts an alignment of the Strix occidentalis caurina control regions 1 and 2. Panel B displays an alignment of the Strix varia control regions 1 and 2 . The numerical coordinates at the top of each panel correspond to the coordinates of the alignment. Black rectangles for each control region denote continuous sequence, whereas intervening horizontal lines denote gaps in the alignment. The sequence identity rectangle is green at full height when there is agreement between the sequences, yellow at less than full height when the sequences disagree, and flat in gap regions. The location of the goose hairpin sequence in each control region is annotated in blue. The alignment locations of the primers we developed to amplify control regions 1 and 2 as well as the D16 primer used by Barrowclough et al. (1999) to amplify a portion of control region 1 are annotated in reddish purple.
$A^{\text {coordinates } 1}$
500
1,000
$1,5,00$
2,000
2,466
S. occid. CR1
-goose hairpin 1

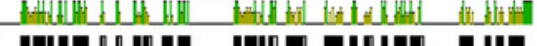

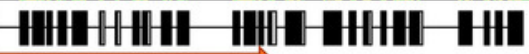
CR1-F1 primer-7 CR1-R2 primer-V V-D16 primer

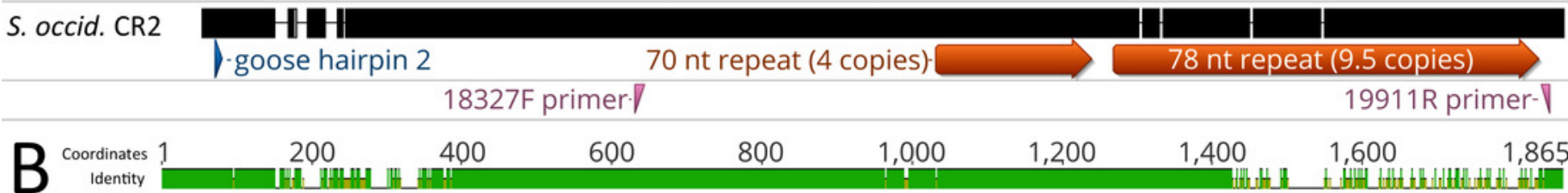
S. varia CR1
goose hairpin $1 \quad$ CR1-F1 primer $\nabla$ CR1-R2 primer-V
S. varia $\mathrm{CR} 2$

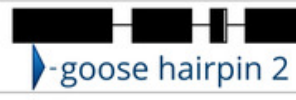
$78 \mathrm{nt}$ repeat (5.2 copies)
19088R primer- $\nabla \quad \nabla$-final-CR2F primer 


\section{Figure 3}

Alignment of Strix occidentalis caurina control regions 1 and 2 with those of Strix varia.

Panel A depicts an alignment of the Strix occidentalis caurina control region 1 with that of Strix varia. Panel B displays an alignment of the Strix occidentalis caurina control region 2 with that of Strix varia. The numerical coordinates at the top of each panel correspond to the coordinates of the alignment. Black rectangles for each control region denote continuous sequence, whereas intervening horizontal lines denote gaps in the alignment. The sequence identity rectangle is green at full height when there is agreement between the sequences, yellow at less than full height when the sequences disagree, and flat in gap regions. The location of the goose hairpin sequence in each control region is annotated in blue. The alignment locations of the primers we developed to amplify control regions 1 and 2 as well as the D16 primer used by Barrowclough et al. (1999) to amplify a portion of control region 1 are annotated in reddish purple. The annotation of primer final-CR2F is elongated as it is situated across a gap region in the alignment.

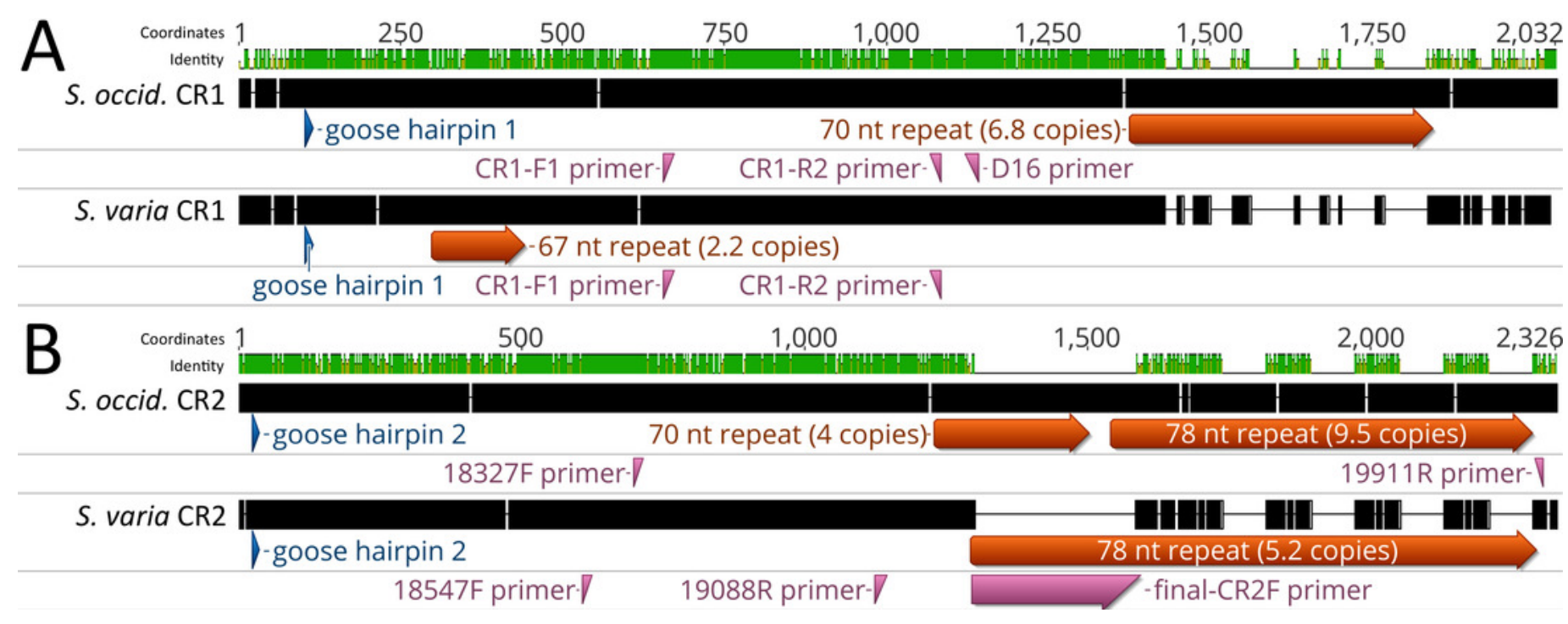

